\title{
An Infrared Investigation of Volatiles in Comet 21P/Giacobini-Zinner
}

\author{
H. A. Weaver \\ Center for Astrophysical Sciences, Department of Physics and Astronomy, The Johns Hopkins University, \\ 3400 North Charles Street, Baltimore, Maryland 21218-2695 \\ E-mail: weaver@pha.jhu.edu \\ G. Chin
}

Laboratory for Extraterrestrial Physics, Planetary Systems Branch, NASA Goddard Space Flight Center, Greenbelt, Maryland 20771

D. Bockelée-Morvan and J. Crovisier

Observatoire de Meudon-Paris, 5 Place Jules Janssen, F-92195 Meudon Cedex, France

T. Y. Brooke

Jet Propulsion Laboratory, MS 169-237, 4800 Oak Grove Drive, Pasadena, California 91109

D. P. Cruikshank

NASA Ames Research Center, MS 245-6, Moffett Field, California 94035-1000

T. R. Geballe

Gemini Observatory, 670 N. A'ohoku Place, Hilo, Hawaii 96720

S. J. Kim

Institute of Natural Sciences, Kyunghee University, Yongin, Kyunggido 449-701 Korea

and

R. Meier

Institute for Astronomy, University of Hawaii, 2680 Woodlawn Drive, Honolulu, Hawaii 96822

Received April 27, 1999; revised August 12, 1999

We obtained high resolution $(\lambda / \delta \lambda \sim 10,000-20,000)$ infrared (IR) spectra of Comet 21P/G iacobini-Zinner (GZ) at five different wavelengths between 1.9 and $5.0 \mu \mathrm{m}$ during 25-29 October 1998 using C SHE LL at the NASA Infrared Telescope F acility (IRTF) on Mauna $\mathrm{Kea}$. We also obtained a moderate resolution $(\lambda / \delta \lambda \sim 680)$ spectrum covering the wavelength range from 3.082 to $3.720 \mu \mathrm{m}$ on 29 October 1998 using C GS4 at the U nited Kingdom Infrared Telescope (UKIRT) on Mauna K ea. Fiverovibrational lines in three different vibrational bands of $\mathrm{H}_{2} \mathrm{O}$ were detected in the CSHELL spectra. Assuming that the rotational temperature was $\sim 50 \mathrm{~K}$, we derive a $\mathrm{H}_{2} \mathrm{O}$ production rate of $\sim 2-3 \times 10^{28}$ molecules $\mathrm{s}^{-1}$, which is $\sim 2$ times smaller than the value derived from nearly simultaneous radio observations of $\mathrm{OH}$. A fter continuum subtraction, the CGS4 spectrum displays significant excess flux that we attribute mainly to $\mathrm{CH}_{3} \mathrm{OH}$ fluorescence, and we derive that the $\mathrm{CH}_{3} \mathrm{OH}$ production rate was $\sim 2.7 \times 10^{26}$ molecules $\mathrm{s}^{-1}$. The corresponding $\mathrm{CH}_{3} \mathrm{OH} / \mathrm{H}_{2} \mathrm{O}$ relative abundance is $\sim 0.9-1.4 \%$, which falls within the range of values observed in other comets, albeit at the low end. The C G S4 spectrum also has significant excess flux near $3.43 \mu \mathrm{m}$ that is not explained by our $\mathrm{CH}_{3} \mathrm{OH}$ fluorescence model; a similar feature has been observed in several other comets, but its origin remains a mystery. We did not detect any excess emission near $3.28 \mu \mathrm{m}$, where some comets show a feature that may be associated with polycyclic aromatic hydrocarbons. We also searched for emissions from $\mathrm{C}_{2} \mathrm{H}_{6}, \mathrm{CO}, \mathrm{HCN}, \mathrm{C}_{2} \mathrm{H}_{2}$, and $\mathrm{H}_{2} \mathrm{CO}$ but did not detect any of these molecules. The $3 \sigma$ upper limits for the abundances relative to $\mathrm{H}_{2} \mathrm{O}$ are $0.05-0.08 \%$ for $\mathrm{C}_{2} \mathrm{H}_{6}, 2-3 \%$ for $\mathrm{CO}, 0.2-0.3 \%$ for $\mathrm{HCN}, 0.3-0.4 \%$ for $\mathrm{C}_{2} \mathrm{H}_{2}$, and $0.5-0.8 \%$ for $\mathrm{H}_{2} \mathrm{CO}$, assuming that all species are parent molecules and that their rotational temperature in the coma is $50 \mathrm{~K}, \mathrm{C}_{2} \mathrm{H}_{6}$ is depleted by a factor of $\sim 15$ or more compared to its relative abundance in Comets Hale-Bopp 
(C/1995 01) and Hyakutake (C/1996 B2); this depletion is similar to that observed for $C_{2}$ and $C_{3}$ from optical observations of $G Z$ (A'H earn et al. 1995) and suggests that the formation of volatile carbon-chain molecules was inhibited in $\mathrm{GZ}$. We are unable to find any clear correlation between the $\mathrm{C}_{2} \mathrm{H}_{6}$ and the $\mathrm{C}_{2}$ and $\mathrm{C}_{3}$ abundances in a sample of nine other comets, assuming that the residual emission near $3.35 \mu \mathrm{m}$ in moderate resolution spectra of seven of the comets provides an accurate indicator of the $\mathrm{C}_{2} \mathrm{H}_{6}$ abundance. $\mathrm{H}$ owever, this latter assumption is questionable and highlights the need to obtain high spectral resolution data in order to make accurate abundance measurements of $\mathrm{C}_{2} \mathrm{H}_{6}$. (c) 1999 A cademic Press

Key Words: comets; composition.

\section{INTRODUCTION}

21P/Giacobini-Zinner (GZ) is a short-period (6.6 yr), Jupiterfamily comet that has been in a relatively stable orbit since its discovery in 1900. As one of the brighter comets in this dynamical family, GZ has been extensively observed throughout this century (see Sekanina 1985). Interest in this comet was especially heightened in 1985 when the Third International Sun-Earth Explorer (ISEE-3) was retargeted and renamed the International Comet Explorer, or ICE, to fly through the tail of GZ at a distance of $\sim 7800 \mathrm{~km}$ from the nucleus. ${ }^{1}$ In support of the flyby mission, an intensive observational campaign was mounted to observe GZ at ultraviolet (UV; McFadden et al. 1987), optical (Cochran and Barker 1987, Schleicher et al. 1987), infrared (IR; Telesco et. al. 1986, Hayward and Grasdalen 1987, Hanner et al. 1992), and radio (Gérard et al. 1988) wavelengths. The spacecraft and campaign results, together with earlier observations, have provided a particularly rich database for GZ and formed an excellent foundation for further studies during its recent 1998 apparition, at which time the dramatically improved technical capabilities at IR and radio wavelengths could be applied.

In their survey of 85 comets, A'Hearn et al. (1995) describe GZ as the "prototypical depleted comet." GZ has $\mathrm{C}_{2}$ and $\mathrm{C}_{3}$ abundances, relative to $\mathrm{H}_{2} \mathrm{O}$, that are $\sim 10$ times smaller than the values measured in most comets, which define the "normal" cometary composition. About one-half of the Jupiter-family comets in the survey exhibited similar depletions, and A'Hearn et al. hypothesized that the depletion is associated with formation in the Kuiper Belt. It is currently thought that most comets, including some currently in the Jupiter family, formed in the vicinity of the giant planets, were ejected into the Oort Cloud, and were subsequently reintroduced into the inner Solar System by various types of gravitational perturbations. Any compositional differences between the Kuiper Belt and Oort Cloud comets might then be reasonably attributed to their different formation locations in the solar nebula.

While the results from A'Hearn et al. certainly represent an important advance in our understanding of comets as a popula-

\footnotetext{
${ }^{1}$ A number of ICE papers on GZ appear in the March and April 1986 issues of Geophysical Research Letters and in the 18 April 1986 issue of Science.
}

tion, their data only indirectly provided information on cometary nuclei because the observed species have an unknown progeny (i.e., the reactions that produce $\mathrm{C}_{2}$ and $\mathrm{C}_{3}$ in the coma are not yet well understood). Furthermore, the $\mathrm{CN}$ abundance in $\mathrm{GZ}$ was normal, leaving open the possibility that other carbon-bearing molecules in the nucleus may not be depleted. One would really like to measure the abundances of the parent molecules that sublime from the nucleus, thus providing direct information on the formation conditions of comets and the physical and chemical state of the solar nebula. Since measurements at infrared wavelengths provide the possibility of detecting various parent molecules via fluorescence in their strong rovibrational transitions (Yamamoto 1982, Crovisier and Encrenaz 1983, Weaver and Mumma 1984), we mounted an observational campaign to investigate the IR spectrum of GZ during late October 1998, when the activity of the comet was expected to peak. Our results, including the first direct detection of $\mathrm{H}_{2} \mathrm{O}$ in GZ, the detection of $\mathrm{CH}_{3} \mathrm{OH}$, and sensitive searches for $\mathrm{C}_{2} \mathrm{H}_{6}$ and $\mathrm{CO}$, are described in this paper.

We note that previous IR and radio detections of parent molecules have generally been limited to bright, long-period comets. Our investigation of volatiles in GZ is an attempt to extend parent molecular searches to the much fainter, shortperiod comets, which are thought to be remnants of the Kuiper Belt. Systematic compositional comparisons of Oort Cloud and Kuiper Belt comets will not be possible until parent molecules can be probed in many objects of both classes.

\section{OBSERVATIONS}

Our IR observations of Comet GZ were made from the NASA Infrared Telescope Facility (IRTF) and the United Kingdom Infrared Telescope (UKIRT), both of which are part of the Mauna Kea Observatories in Hawaii. We observed shortly after sunset each evening during the period UT 25-29 October 1998. Perihelion was on 21 November, and we chose to observe about one month earlier because observations in 1985 indicated that the comet's activity peaked at that time. The IRTF observations covered small wavelength intervals $(\sim 0.005-0.0125 \mu \mathrm{m})$ centered at five specific wavelengths (Table I). The UKIRT observations covered the entire spectral region from 3.08 to $3.72 \mu \mathrm{m}$.

During our observations, the comet's heliocentric distance $(r)$ ranged from 1.08 to $1.11 \mathrm{AU}$, the geocentric distance $(\Delta)$ ranged from 0.93 to $0.96 \mathrm{AU}$, the solar phase angle (Earth-comet-Sun) ranged from $57^{\circ}$ to $58^{\circ}$, the heliocentric radial velocity $(\dot{r})$ ranged from -8.6 to $-7.3 \mathrm{~km} \mathrm{~s}^{-1}$, and the geocentric radial velocity $(\dot{\Delta})$ ranged from -8.8 to $-8.2 \mathrm{~km} \mathrm{~s}^{-1}$. Unfortunately, $\dot{\Delta}$ remained relatively small for a couple of months on either side of perihelion, and this severely compromised our investigation of $\mathrm{CO}$ and eliminated the possibility of searching for $\mathrm{CH}_{4}$, because of strong interference from the corresponding transitions in the terrestrial atmosphere. Typically we would observe a calibration star between UT 3:30 and 4:30 (i.e., between 5:30 pm and 6:30 pm local time) and then observe the comet for the 
TABLE I

Log of 21P/G iacobini-Zinner Observations

\begin{tabular}{|c|c|c|c|c|c|c|c|c|c|}
\hline $\begin{array}{l}\text { Date } \\
\text { (UT 199 }\end{array}$ & $\begin{array}{l}\text { Start } \\
8 \mathrm{HH}: \mathrm{MN}\end{array}$ & $\begin{array}{l}\text { Stop } \\
\text { M) }\end{array}$ & Instrument & Objective & $\begin{array}{l}\text { Grating } \\
\left(\mathrm{cm}^{-1}\right)\end{array}$ & $\begin{array}{l}\text { Setting } \\
(\mu \mathrm{m})\end{array}$ & $\begin{array}{l}\text { Coadded frames } \\
\text { (number) }\end{array}$ & $\begin{array}{l}\text { Exposure time } \\
\text { (minutes) }\end{array}$ & Airmass \\
\hline 25 October & $04: 49$ & $07: 50$ & CSHELL & $\mathrm{C}_{2} \mathrm{H}_{6}$ & 2989 & 3.346 & 170 & 170 & $1.26-4.89$ \\
\hline 26 October & $06: 14$ & $07: 17$ & CSHELL & $\mathrm{CO}, \mathrm{H}_{2} \mathrm{O}$ & 2150 & 4.651 & 288 & 48 & $1.81-3.05$ \\
\hline 27 October & $05: 37$ & $07: 15$ & CSHELL & $\mathrm{H}_{2} \mathrm{O}$ & 2003 & 4.993 & 454 & 76 & $1.51-2.99$ \\
\hline 28 October & $04: 35$ & $06: 53$ & CSHELL & $\mathrm{HCN}, \mathrm{C}_{2} \mathrm{H}_{2}$ & 3303 & 3.028 & 108 & 108 & $1.23-2.46$ \\
\hline 29 October & $04: 44$ & $05: 58$ & CSHELL & $\mathrm{H}_{2} \mathrm{O}$ & 5084 & 1.967 & 22 & 66 & $1.29-1.68$ \\
\hline 29 October & 04:49 & $06: 15$ & CGS4 & $\begin{array}{c}\mathrm{CH}_{3} \mathrm{OH}, \mathrm{H}_{2} \mathrm{CO} \\
\text { organics }\end{array}$ & 2940 & 3.40 & 48 & 32 & $1.28-1.80$ \\
\hline
\end{tabular}

next few hours. A second calibration star was always observed after the cometary observations. The weather conditions were good throughout the period of our observations, with a seeing of $\sim 0^{\prime \prime} .6-0^{\prime \prime} .8$. The column density of atmospheric $\mathrm{H}_{2} \mathrm{O}$ decreased monotonically from $\sim 2.4$ to $\sim 1 \mathrm{~mm}$ of precipitable $\mathrm{H}_{2} \mathrm{O}$ during the course of our observing run. Table I provides a log of our observations.

\subsection{IRTF CSHELL}

Most of our observations were made from the NASA Infrared Telescope Facility (IRTF) using the Cryogenic Echelle Spectrometer (CSHELL; Greene et al. 1993). The resolving power of CSHELL varies from $\sim 5,400$ to $\sim 43,000$ depending on the width of the slit used. We primarily used the $2^{\prime \prime}$ slit for our cometary observations, which yields a resolving power of $\sim 10,000$ for a source filling the slit in the dispersion direction, but our search for $\mathrm{CO}$ on 26 October was made using the $1^{\prime \prime}$ slit to provide better discrimination between the cometary emission and the corresponding terrestrial $\mathrm{CO}$ emission. All of the CSHELL slits extend $\sim 30^{\prime \prime}$ perpendicular to dispersion, and analysis of data along this latter direction can, in principle, provide information on the spatial distribution of the cometary emissions (see Weaver et al. 1999). However, owing to the faintness of GZ, ${ }^{2}$ we decided to nod $10^{\prime \prime}$ along the slit to keep the nucleus on the detector at all times to improve the sensitivity. The slit was always oriented with the long dimension, which is the spatial dimension, along the celestial north-south direction. The celestial position angle of the projected solar vector was $\sim 77^{\circ}$, which is nearly due east. Individual CSHELL pixels are square and are $0^{\prime \prime} .2$ on a side, which projected to $\sim 135 \mathrm{~km}$ at the comet during our observations.

The spectral coverage for a given CSHELL grating setting is only $\sim 2.5 \times 10^{-3} \lambda$, where $\lambda$ is the central wavelength in the spectrum. This limited free spectral range, and the faintness of the comet, meant that we had to focus on a single scientific objective each evening (see Table I).

All of the CSHELL observations were obtained in ABBA mode, where " $A$ " and "B" refer to integrations having identical exposure times but with a telescope displacement of $10^{\prime \prime}$ in the north-south direction. Thus, the nucleus of the comet would appear centered in the aperture at one position on the detector for the A integration and then would be moved $10^{\prime \prime}$ along the slit, but still centered in the aperture, for the B integration. Many ABBA sequences were obtained each evening (Table I). The "B" spectral images were coadded and subtracted from the coadded "A" spectral images to obtain a single net spectral image for each date. For the CSHELL observations taken on 26, 27, and 29 October, clear evidence of the cometary continuum could be seen in the net spectral images as positive and negative "streaks" along the dispersion direction, which is the row direction on the IR arrays, separated from each other by $10^{\prime \prime}$. Detection of the cometary continuum on 25 and 28 October was marginal, being seen only in the extracted spectra produced by adding data in 20 rows, which corresponds to an effective aperture height of $4^{\prime \prime}$. None of the spectral lines discussed in this paper were obvious in the final spectral images, but statistically significant features were detected in the spectral extractions in several cases (see Table II).

The cometary emission from parent molecules at $\sim 10^{\prime \prime}$ from the nucleus is expected to be lower by about an order of magnitude compared to its value for a nucleus-centered observation. Even for $\mathrm{CO}$ emission, which comes partly from an extended source in the coma, the signal at an offset of $10^{\prime \prime}$ should not be more than $\sim 30 \%$ of its value in an aperture centered on the nucleus (see Weaver et al. 1999). Since we could only barely detect the cometary emission at the nucleus, we neglected any contamination of the A beam by the B beam, and vice-versa. However, we note that our technique for extracting spectra could underestimate significantly the signal from any daughter or granddaughter species. $^{3}$

Observations of calibration stars, flat-fields, and darks were also obtained at each CSHELL grating setting. All absolute fluxes for the CSHELL data presented here were derived from observations of the star HR 6707 (v Her, spectral type F2II, $T_{\text {eff }} \sim 7000 \mathrm{~K}$ ), which is a calibration standard at both the IRTF and the United Kingdom Infrared Telescope (UKIRT). The

\footnotetext{
${ }^{3}$ These are species that are produced in the coma, rather than coming directly from the nucleus. 
TABLE II

Fluxes and Production Rates for 21P/G iacobini-Zinner Observations

\begin{tabular}{|c|c|c|c|c|c|c|c|c|}
\hline $\begin{array}{c}\text { Date } \\
\text { (UT 1998) }\end{array}$ & Line or band $\mathrm{ID}^{a}$ & $\begin{array}{l}\text { Frequency } \\
\left(\mathrm{cm}^{-1}\right)\end{array}$ & $\begin{array}{l}\mathrm{FOV}^{c} \\
(\operatorname{arcsec})\end{array}$ & $\begin{array}{l}\text { Line or band flux }{ }^{d} \\
\left(10^{-18} \mathrm{~W} \mathrm{~m}^{-2}\right)\end{array}$ & $\begin{array}{l}\mathrm{g} \text { factor }^{e} \\
\left(10^{-5} \mathrm{~s}^{-1}\right)\end{array}$ & $\begin{array}{l}\text { Production rate } \\
\quad\left(10^{28} \mathrm{~s}^{-1}\right)\end{array}$ & $\begin{array}{c}\text { Abundance }{ }^{g} \\
(\%)\end{array}$ & $\begin{array}{c}\text { Continuum flux } \\
\left(10^{-15} \mathrm{~W} \mathrm{~m}^{-2} \mu \mathrm{m}^{-1}\right)\end{array}$ \\
\hline 25 October & $\mathrm{C}_{2} \mathrm{H}_{6}{ }^{\mathrm{r}} \mathrm{Q}_{0}$ & 2986.749 & $2 \times 4$ & $\leq 0.37$ & 5.6 & $\leq 0.0015$ & $\leq 0.05-0.08$ & $3.5 \pm 1.8$ \\
\hline 26 October & CO R0 & 2147.082 & $1 \times 4$ & $\leq 1.3$ & 1.1 & $\leq 0.064$ & $\leq 2.1-3.2$ & $3.8 \pm 1.9$ \\
\hline 26 October & CO R1 & 2150.856 & $1 \times 4$ & $\leq 2.2$ & 1.8 & $\leq 0.068$ & $\leq 2.3-3.4$ & $3.8 \pm 1.9$ \\
\hline 26 October & $\begin{array}{c}\mathrm{H}_{2} \mathrm{O} 2_{21}-1_{10} \\
v_{1}-v_{2}\end{array}$ & 2148.187 & $1 \times 4$ & $1.0 \pm 0.7$ & 0.011 & $5.3 \pm 2.6$ & 100 & $3.8 \pm 1.9$ \\
\hline 26 October & $\begin{array}{c}\mathrm{H}_{2} \mathrm{O} 1_{11}-1_{10} \\
\nu_{3}-\nu_{2}\end{array}$ & 2151.194 & $1 \times 4$ & $1.3 \pm 0.7$ & 0.057 & $1.2 \pm 0.6$ & 100 & $3.8 \pm 1.9$ \\
\hline 27 October & $\begin{array}{c}\mathrm{H}_{2} \mathrm{O} 2_{12}-3_{03} \\
v_{1}-v_{2}\end{array}$ & 2002.999 & $2 \times 4$ & $3.0 \pm 1.5$ & 0.024 & $4.3 \pm 2.2$ & 100 & $12 \pm 4.2$ \\
\hline 27 October & $\begin{array}{c}\mathrm{H}_{2} \mathrm{O} 1_{01}-2_{12} \\
v_{1}-v_{2}\end{array}$ & 2003.391 & $2 \times 4$ & $4.2 \pm 2.1$ & 0.058 & $2.4 \pm 1.2$ & 100 & $12 \pm 4.2$ \\
\hline 28 October & HCN P3 & 3302.546 & $2 \times 4$ & $\leq 0.90$ & 3.3 & $\leq 0.0054$ & $\leq 0.18-0.27$ & $2.2 \pm 1.1$ \\
\hline 28 October & $\mathrm{C}_{2} \mathrm{H}_{2} \mathrm{R} 3$ & 3304.167 & $2 \times 4$ & $\leq 0.59$ & 1.6 & $\leq 0.0083$ & $\leq 0.28-0.42$ & $2.2 \pm 1.1$ \\
\hline 29 October & $\begin{array}{c}\mathrm{H}_{2} \mathrm{O} 2_{02}-3_{03} \\
v_{1}+v_{2}+v_{3}-v_{1}\end{array}$ & 5083.937 & $2 \times 4$ & $0.43 \pm 0.22$ & 0.0024 & $2 \pm 1$ & 100 & $10 \pm 3.5$ \\
\hline 29 October & $\mathrm{CH}_{3} \mathrm{OH} v_{3}$ & $2825-2874$ & $1.2 \times 2.4$ & $10 \pm 3$ & 15 & $0.027 \pm 0.008$ & $0.9-1.4$ & $2.0 \pm 0.2$ \\
\hline 29 October & $3.43 \mu \mathrm{m}$ & $2857-2985$ & $1.2 \times 2.4$ & $21 \pm 7$ & - & - & - & $1.8 \pm 0.2$ \\
\hline 29 October & $\mathrm{H}_{2} \mathrm{CO} v_{1}, v_{5}$ & $2778-2801$ & $1.2 \times 2.4$ & $\leq 6.6$ & 18 & $\leq 0.015$ & $\leq 0.5-0.8$ & $2.1 \pm 0.2$ \\
\hline
\end{tabular}

${ }^{a}$ ID: "3.43 $\mu \mathrm{m}$ " refers to the residual emission feature centered at $3.43 \mu \mathrm{m}$.

${ }^{b}$ Frequency: rest values.

${ }^{c}$ FOV: effective aperture size.

${ }^{d}$ Fluxes: errors include absolute calibration and continuum uncertainties.

${ }^{e} \mathrm{~g}$ factor: line values assume $T_{\text {rot }}=50 \mathrm{~K} ; \mathrm{H}_{2} \mathrm{CO}$ value is $25 \%$ of the sum of the $v_{1}$ and $v_{5}$ band $\mathrm{g}$ factors, which is the fraction within the integration limits.

${ }^{f}$ Production rate: Errors do not include the uncertainty in $T_{\text {rot }}$; upper limits are $3 \sigma$ values.

${ }^{g}$ Abundance: relative to $\mathrm{H}_{2} \mathrm{O}$, assuming $\mathrm{Q}_{\mathrm{H}_{2} \mathrm{O}}=2-3 \times 10^{28}$ molecules s${ }^{-1}$, which is our best estimate (see text for further discussion).

magnitudes of HR 6707 are 3.21, 3.16, 3.18, and 3.17 at $\mathrm{K}$ $\left(\lambda_{\text {eff }}=2.21 \mu \mathrm{m}, \Delta \lambda=0.39 \mu \mathrm{m}\right), \mathrm{L}\left(\lambda_{\text {eff }}=3.50 \mu \mathrm{m}, \Delta \lambda=\right.$ $0.61 \mu \mathrm{m}), \mathrm{L}^{\prime}\left(\lambda_{\text {eff }}=3.78 \mu \mathrm{m}, \Delta \lambda=0.59 \mu \mathrm{m}\right)$, and $\mathrm{M}\left(\lambda_{\text {eff }}=\right.$ $4.85 \mu \mathrm{m}, \Delta \lambda=0.62 \mu \mathrm{m})$, respectively, with an error of $\sim 0.01$ mag for each. For the stellar observations using CSHELL, we observed through the $1^{\prime \prime}, 2^{\prime \prime}$, and $4^{\prime \prime}$-wide slits to gauge the effect of seeing and tracking on the observed signal. Typically, the flux from the star in a $4^{\prime \prime} \times 4^{\prime \prime}$ aperture was about twice as large as that in a $1^{\prime \prime} \times 4^{\prime \prime}$ aperture and $\sim 20-50 \%$ larger than the flux measured in a $2^{\prime \prime} \times 4^{\prime \prime}$ aperture. We always used the stellar flux in a $4^{\prime \prime} \times 4^{\prime \prime}$ slit to define the absolute calibration. We did not make any aperture loss correction for the cometary data, even though the latter were usually taken through a $2^{\prime \prime}$ slit $^{4}$ because the spatial brightness distribution for a comet is more diffuse than that from the star, and no correction is required in the limiting case of an emission that uniformly fills the extraction aperture. Furthermore, unlike the stellar observations which were made during the day, the cometary observations were made at night when an optical image of the comet could be viewed through a coaligned CCD camera while taking its IR spectrum, and active guiding was used to keep the comet centered in the CSHELL slit to an accuracy comparable to the size of the seeing disk. Using a model that takes into account the expected spatial distribution of the cometary emission, we estimate that the signal collected in a $2^{\prime \prime} \times 4^{\prime \prime}$ aperture would decrease by only $\sim 5 \%$ when the nucleus is displaced by $0^{\prime \prime} .6$ in the dispersion direction from the slit center. For a $1^{\prime \prime} \times 4^{\prime \prime}$ aperture, a $0^{\prime \prime} .6$ displacement in the dispersion direction would decrease the observed flux by $\sim 25 \%$. The decrease in the aperture transmittance due to the finite size of the point spread function of the telescope is estimated to be $\sim 2 \%$ for the cometary data taken through a $2^{\prime \prime} \times 4^{\prime \prime}$ slit and $\sim 8 \%$ for the cometary data taken through a $1^{\prime \prime} \times 4^{\prime \prime}$ slit. In summary, the CSHELL cometary fluxes quoted in this paper might be systematically underestimated, particularly when using the $1^{\prime \prime}$ slit, because we have neglected aperture-loss corrections, but this error is generally not expected to be the dominant error affecting the observed absolute fluxes, as discussed in later sections. Furthermore, since this systematic error applies to all parent molecular emissions, our relative abundances should not be affected. We note also that both the CSHELL and CGS4 observations (discussed below) were made with similar-sized apertures, so any loss factor that applies to the CSHELL results probably also must be applied to the CGS4 results.

To convert the observed cometary signals to absolute fluxes for the CSHELL data, we did not follow the standard technique

\footnotetext{
${ }^{5}$ Note that similar motions perpendicular to dispersion cause essentially no loss in flux as we integrate over $4^{\prime \prime}$ in that direction. 
of dividing the observed cometary spectrum by the observed stellar spectrum and then multiplying by the absolute stellar flux. That procedure produces artifacts near strong terrestrial features, especially when the airmass and atmospheric conditions are not perfectly matched for the star and comet. Instead, we first computed a model stellar spectrum, including the atmospheric absorption calculated using the Fast Atmospheric Signature Code (FASCOD2) radiative transfer software (Clough et al. 1986) and the High-resolution Transmission molecular Absorption (HITRAN) database (Rothman et al. 1992). Then we computed "inverse sensitivity factors" at each data point by fitting a smoothly varying spline function to the ratio of the model and observed stellar spectrum. ${ }^{6}$ Multiplying the observed cometary spectrum by the inverse sensitivity factors gives the absolutely calibrated cometary spectrum. Next, we divided the absolutely calibrated cometary spectrum by a model atmospheric transmittance spectrum at the same airmass and resolution as the cometary spectrum, fit a smoothly varying spline function to that ratio in the regions of good atmospheric transmittance, and multiplied the atmospheric transmittance spectrum by this spline function to obtain an estimate of the observed cometary continuum (i.e., the cometary continuum at the telescope). Subtraction of the latter from the full cometary spectrum yields an emission spectrum, from which integrated line fluxes were calculated. Division of the integrated line flux by the monochromatic atmospheric transmittance at the center of the observed line then gives the integrated line flux at the top of the atmosphere. Similarly, division of the estimated cometary continuum at the telescope by the model atmospheric transmittance gives the estimated cometary continuum at the top of the atmosphere. Examples of the estimated cometary continuum spectra at the telescope, and of the emission spectra, are shown later in the paper.

The frequency scale for each CSHELL spectrum was determined by identifying several atmospheric absorption lines in the spectrum and calculating a linear dispersion formula that fit the known absolute positions of the atmospheric lines in a least-squares sense. When the continuum was too weak to identify absorption lines clearly (e.g., for the cometary spectra taken on 25 and 28 October), we fit the corresponding atmospheric emission lines in the "A" or "B" frame. We estimate that the dispersion relation so determined was accurate to $\sim 1$ pixel, which corresponds approximately to $9.6 \times 10^{-6} v \mathrm{~cm}^{-1}$ where $v$ is the central frequency $\left(\mathrm{cm}^{-1}\right)$ for a particular grating setting. ${ }^{7}$ For the data taken on 27 October, there was only one atmospheric absorption/emission line in the entire spectrum. In this particular case, we derived a dispersion relation using this single atmospheric line and the strongest observed line in the cometary emission spectrum, which we assumed to be the $1_{01}-2_{12}$ line in the $v_{1}-v_{2}$ band of $\mathrm{H}_{2} \mathrm{O}$. If our identification of the cometary

\footnotetext{
${ }^{6}$ Use of the spline function avoids singularities in the regions of poor atmospheric transmittance.

${ }^{7}$ The dispersion in velocity space is $\sim 2.9 \mathrm{~km} \mathrm{~s}^{-1}$ pixel $^{-1}$.
}

$\mathrm{H}_{2} \mathrm{O}$ emission line is correct, then the frequency scale for the 27 October data should have an accuracy of $\sim 2$ pixels.

\subsection{UKIRT CGS4}

On UT 29 October 1998 we also obtained a spectrum of GZ using the Cooled Grating Spectrometer 4 (CGS4) at the UKIRT and covering the wavelength range 3.08-3.72 $\mu \mathrm{m}$. Individual CGS4 pixels are square and are $0^{\prime \prime} .61$ on a side, which projected to $\sim 410 \mathrm{~km}$ at the comet during our observation. The 40 lines $\mathrm{mm}^{-1}$ grating was used along with a $1^{\prime \prime} .2$ wide slit, resulting in a resolution of $0.0050 \mu \mathrm{m}$, corresponding to $\lambda / \delta \lambda \sim 680$ at $3.40 \mu \mathrm{m}$. The UKIRT data were obtained in ABBA mode, using nodding along the slit, which was oriented east-west. Spectra were obtained with nods of both $7^{\prime \prime} .32$ and $14^{\prime \prime} .64$. The four positive and negative rows with the largest signals were extracted from the final coadded spectral image and combined, resulting in an effective aperture size of $1^{\prime \prime} .2 \times 2^{\prime \prime} .4(\mathrm{NS} \times \mathrm{EW})$. Comparison of the extracted spectra with the smaller and larger nods showed little or no reduction of signal in the former due to the subtraction of extended cometary emission. They were averaged to provide the final spectrum.

Spectra of HR 7272 (spectral type G1V, $T_{\text {eff }}=5900 \mathrm{~K}$ ) at two airmasses were obtained for the purpose of flux calibration and removal of telluric and instrumental spectral features. The star was assumed to have an L magnitude of 5.26, using the color relations in Tokunaga (2000). From the intensity profile of the star along the slit, we estimate that the extracted spectrum contains $50 \pm 5 \%$ of the stellar flux incident on the slit. Wavelength calibration was obtained from an argon lamp observed in second order and is accurate to better than $0.0010 \mu \mathrm{m}$. The flux-calibrated spectrum of GZ presented in this paper was rebinned to a resolution of $0.01 \mu \mathrm{m}$, corresponding to $\lambda / \delta \lambda \sim 340$ at $3.40 \mu \mathrm{m}$.

\section{RESULTS AND DISCUSSION}

\section{1. $\mathrm{H}_{2} \mathrm{O}$}

Water had never been directly detected in GZ, although its presence has been inferred from UV and radio observations of OH (McFadden et al. 1987, Schleicher et al. 1987, Gérard et al. 1988). These previous studies of $\mathrm{OH}$ demonstrated conclusively that $\mathrm{H}_{2} \mathrm{O}$ is the dominant volatile constituent in GZ, a property that $\mathrm{GZ}$ probably shares with all other comets. Since $\mathrm{H}_{2} \mathrm{O}$ is so much more abundant than other cometary volatiles, its sublimation is primarily responsible for the observed activity of GZ, at least for $r \leq 3 \mathrm{AU}$ when temperatures at the surface of the nucleus are expected to exceed $\sim 150 \mathrm{~K}$. We detected several IR transitions of $\mathrm{H}_{2} \mathrm{O}$ during our CSHELL observations on 26, 27, and 29 October.

On 29 October, we searched for $\mathrm{a}_{2} \mathrm{O}$ line in the $v_{1}+v_{2}+$ $\nu_{3}-v_{1}$ band near $2 \mu \mathrm{m}$ and found a significant emission feature at exactly the predicted Doppler-shifted position (Fig. 1). Although the peak flux has a signal-to-noise ratio of only $\sim 3-4$, 


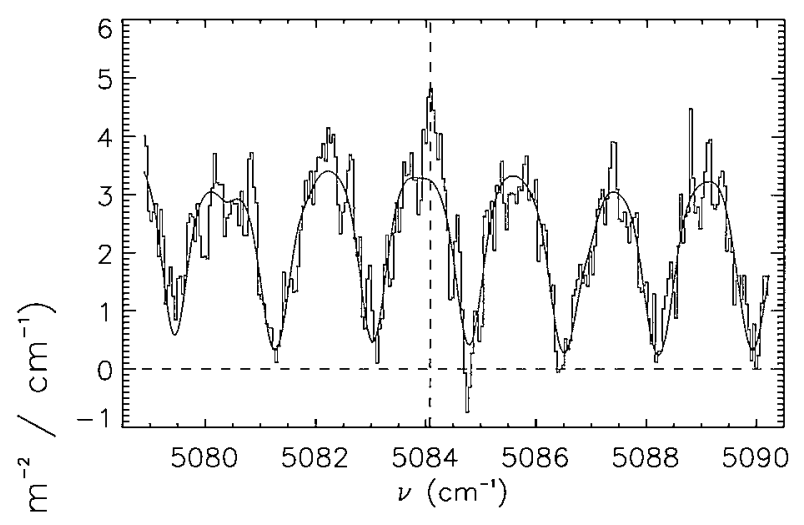

3

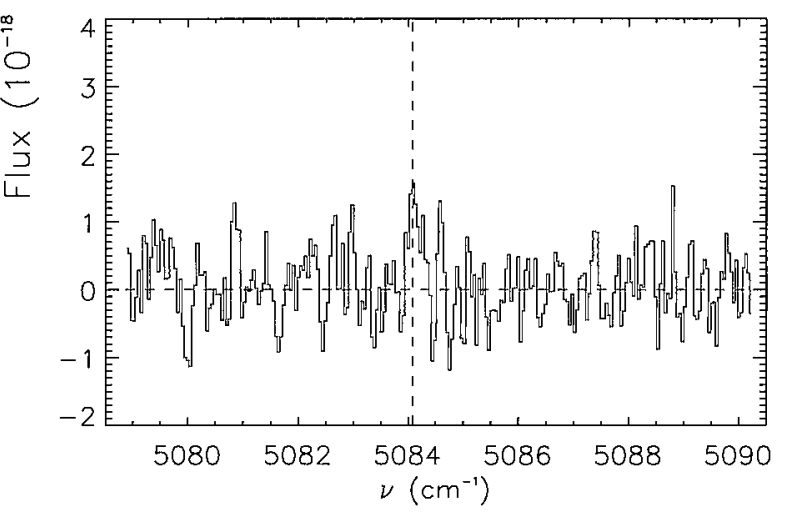

FIG. 1. The top panel shows a spectrum of Comet 21P/Giacobini-Zinner taken on UT 29 October 1998 using CSHELL at the NASA IRTF. The predicted location of the $2_{02}-3_{03} \mathrm{H}_{2} \mathrm{O}$ line in the $v_{1}+v_{2}+v_{3}-v_{1}$ band is shown by the dashed vertical line. The solid curve is a model for the observed cometary continuum, including the atmospheric absorption. Subtraction of the estimated continuum from the observed spectrum yields the emission spectrum shown in the bottom panel. The integrated flux in the $\mathrm{H}_{2} \mathrm{O}$ line is detected with a signalto-noise ratio of $\sim 8$.

the feature has a spectral shape consistent with that expected for a real cometary emission observed through the $2^{\prime \prime}$ slit (the slit width is 10 pixels), and the signal-to-noise ratio for the lineintegrated flux is $\sim 8$. The many absorption features in the spectrum are due to an overtone band of $\mathrm{CO}_{2}$ in the terrestrial atmosphere, and no cometary emission can be detected in the cores of these lines because the transmittance goes to zero there. The identified line $\left(2_{02}-3_{03}\right)$ is expected to be the strongest one in the band and is the only reasonably strong $\mathrm{H}_{2} \mathrm{O}$ line within the spectral range covered by this grating setting. The success of our observation depended critically on the favorable Doppler shift, which put the cometary emission at a wavelength where the atmospheric transmittance was $\sim 85 \%$. The main source of error in determining the absolute flux of the $\mathrm{H}_{2} \mathrm{O}$ line is the uncertainty in estimating the continuum. By experimenting with various choices for the continuum, we conclude that the emission line flux has an accuracy of $\sim 50 \%$, and the continuum flux uncertainty is $\sim 35 \%$ (see Table II). From the flux for the cometary continuum, we derive Af $\sim 720 \pm 250 \mathrm{~cm}$, where
Af $\rho$ is the product of the grain albedo, the grain filling factor, ${ }^{8}$ and the radius of the equivalent circular aperture used during the observations (A'Hearn et al. 1984). Our value of $A f \rho$ at $\lambda=2 \mu \mathrm{m}$ is somewhat larger than, but comparable to, the values derived from optical observations at $\lambda=4845 \AA$ and $r=1.08$ AU during the 1985 apparition $(A f \rho \sim 420 \mathrm{~cm}$; Schleicher et al. 1987).

Using the absolute flux ( $F$ in $\mathrm{W} \mathrm{m}^{-2}$ ) of the observed $\mathrm{H}_{2} \mathrm{O}$ line at the top of the atmosphere, we calculated the $\mathrm{H}_{2} \mathrm{O}$ production rate $\left(\mathrm{Q}_{\mathrm{H}_{2} \mathrm{O}}\right.$, molecules $\left.\mathrm{s}^{-1}\right)$ according to

$$
\mathrm{Q}_{\mathrm{H}_{2} \mathrm{O}}=\frac{2.48 \times 10^{43} v r^{2} \Delta F}{g v \theta},
$$

where $v$ is the outflow velocity $\left(=0.8 / \sqrt{r} \mathrm{~km} \mathrm{~s}^{-1}\right), g$ is the fluorescence efficiency factor at $r=1 \mathrm{AU}$ (photons $\mathrm{s}^{-1}$ molecule $^{-1}$ ), $v$ is the frequency of the line $\left(\mathrm{cm}^{-1}\right)$, and $\theta$ is the diameter of the equivalent circular aperture (arcsec) used for the spectral extraction. ${ }^{9}$ The above formula will also be used to calculate the other molecular production rates discussed in this paper. The formula ignores the finite lifetime of the molecule, but the effect of including the lifetime is negligible for all the small-aperture observations discussed here.

We use the detailed, multiband excitation model of BockeléeMorvan and Crovisier (1988) to calculate $\mathrm{g}$ factors for the $\mathrm{H}_{2} \mathrm{O}$ lines discussed in this paper. The model has been extended to include more vibrational bands, including all of the bands observed in GZ. The model has previously been applied successfully in the analysis of several medium-to-high resolution cometary spectra showing $\mathrm{H}_{2} \mathrm{O}$ rovibrational lines (e.g., Crovisier et al. 1997). For our analysis of the GZ data, we assume that the population of the rotational levels in the ground vibrational state can be described by a thermal distribution. ${ }^{10}$ Therefore, the only input parameter to the model is the effective rotational temperature of the $\mathrm{H}_{2} \mathrm{O}$ molecules, which we could not independently determine because we only observed one to three lines in any particular vibrational band and their relative intensities are not very sensitive to the rotational temperature. Given that the outside edge of the aperture used for the spectral extraction was only $\sim 1400 \mathrm{~km}$ from the nucleus, we would expect thermalized molecules in that region of the coma to be very cold due to the strong expansion cooling (see Bockelée-Morvan and Crovisier 1987). Even if collisional equilibrium was not achieved because GZ has such

\footnotetext{
${ }^{8}$ The grain filling factor is effectively the optical depth.

${ }^{9}$ We used $3^{\prime \prime} .2$ for the $2^{\prime \prime} \times 4^{\prime \prime}$ case and $1^{\prime \prime} .8$ for the $1^{\prime \prime} \times 4^{\prime \prime}$ case; these were determined from a model analysis in which we calculated which circular aperture gives the same integrated flux as the rectangular apertures employed during the observations.

${ }^{10}$ We also assume that the ortho-to-para ratio (OPR) is the statistical equilibrium value of 3 , which is achieved in thermal equilibrium whenever $T \geq 50 \mathrm{~K}$. This choice is justified if the $\mathrm{H}_{2} \mathrm{O}$ molecules equilibrate at the temperature of the outer surface of the nucleus, since the ratio cannot be changed by coma processes. In any event, our value of $\mathrm{Q}_{\mathrm{H}_{2} \mathrm{O}}$ is not very sensitive to the exact value of the OPR; for example, using OPR $=2.4$ only changes $\mathrm{Q}_{\mathrm{H}_{2} \mathrm{O}}$ by $\sim 6 \%$. See Mumma et al. (1987) for further discussion.
} 
low gas production rates, the rotational temperature of $\mathrm{H}_{2} \mathrm{O}$ is still expected to be low because fast rotational cooling, owing to the large permanent electric dipole moment of $\mathrm{H}_{2} \mathrm{O}$, dominates the radiative pumping induced by infrared flourescence of solar light. We have calculated the evolution of the rotational population distribution with cometocentric distance using the comprehensive model of Bockelée-Morvan (1987) and find that collisional equilibrium should apply within the field-of-view of our observations. Throughout this paper we will calculate production rates using $T_{\text {rot }}=50 \mathrm{~K}$, as this is an appropriate choice for a low-activity comet like GZ (see Bockelée-Morvan and Crovisier 1987).

Assuming that $T_{\text {rot }}$ is $50 \mathrm{~K}$, the nominal $\mathrm{H}_{2} \mathrm{O}$ production rate derived from our observation of the $2_{02}-3_{03}$ line in the $v_{1}+v_{2}+$ $v_{3}-v_{1}$ band is $2.0 \times 10^{28}$ molecules $\mathrm{s}^{-1}$ (Table II). If $T_{\text {rot }}$ is $100 \mathrm{~K}$, then $\mathrm{Q}_{\mathrm{H}_{2} \mathrm{O}}$ increases to $2.4 \times 10^{28}$ molecules s ${ }^{-1}$.

We must also point out that the calculation of $\mathrm{g}$ factors for the $v_{1}+v_{2}+v_{3}-v_{1}$ band of $\mathrm{H}_{2} \mathrm{O}$ might have some systematic error because the intensities of the lines in this "hot" band have never been measured in the laboratory. Our calculations assume that the Einstein A values for the lines in this band are identical to the Einstein A values for the lines in the $v_{2}+v_{3}$ band, an assumption that was used previously by Mumma et al. (1996) in their analysis of the $\mathrm{H}_{2} \mathrm{O}$ lines they observed in Comet Hyakutake (1996 B2) and which gave production rates that were comparable to those derived using other techniques (e.g., observations of $\mathrm{OH})$. A recent ab initio calculation of the intensities of $\mathrm{H}_{2} \mathrm{O}$ lines (Partridge and Schwenke 1997) also gives excellent agreement (within 2\%) with our estimate for the strength of the $2_{02}-3_{03}$ line.

All production rates listed in Table II include a conservative estimate of the total error due to measurement uncertainty and the absolute flux calibration, but they do not include the error associated with the uncertainty in $T_{\text {rot }}{ }^{11}$ Since the dominant error is generally that associated with the continuum removal, the limits given for the production rates listed in Table II should be regarded as plausible ranges for the values and not $1 \sigma$ statistical limits.

The cometary spectra obtained on 26 and 27 October were taken in the 5- $\mu \mathrm{m}$ wavelength region, where the thermal emission from the terrestrial atmosphere is orders of magnitude larger than the terrestrial emission observed in the $2-\mu \mathrm{m}$ region. In order to provide for good subtraction of this high background, we beam-switched every $10 \mathrm{~s}$. The observations on 27 October were taken through the $2^{\prime \prime}$ slit, while a $1^{\prime \prime}$ slit was used on 26 October to provide better discrimination between the cometary and terrestrial lines of $\mathrm{CO}$.

The grating setting used on 27 October covered a spectral range that included two strong lines in the $v_{1}-v_{2}$ band of $\mathrm{H}_{2} \mathrm{O}$. The P10 transition of the $\mathrm{CN}(1,0)$ vibrational band (the rest frequency is $2003.07 \mathrm{~cm}^{-1}$ ) also lies within this wavelength

\footnotetext{
${ }^{11}$ Note, however, that the production rates based on band intensities are independent of $T_{\text {rot }}$.
}

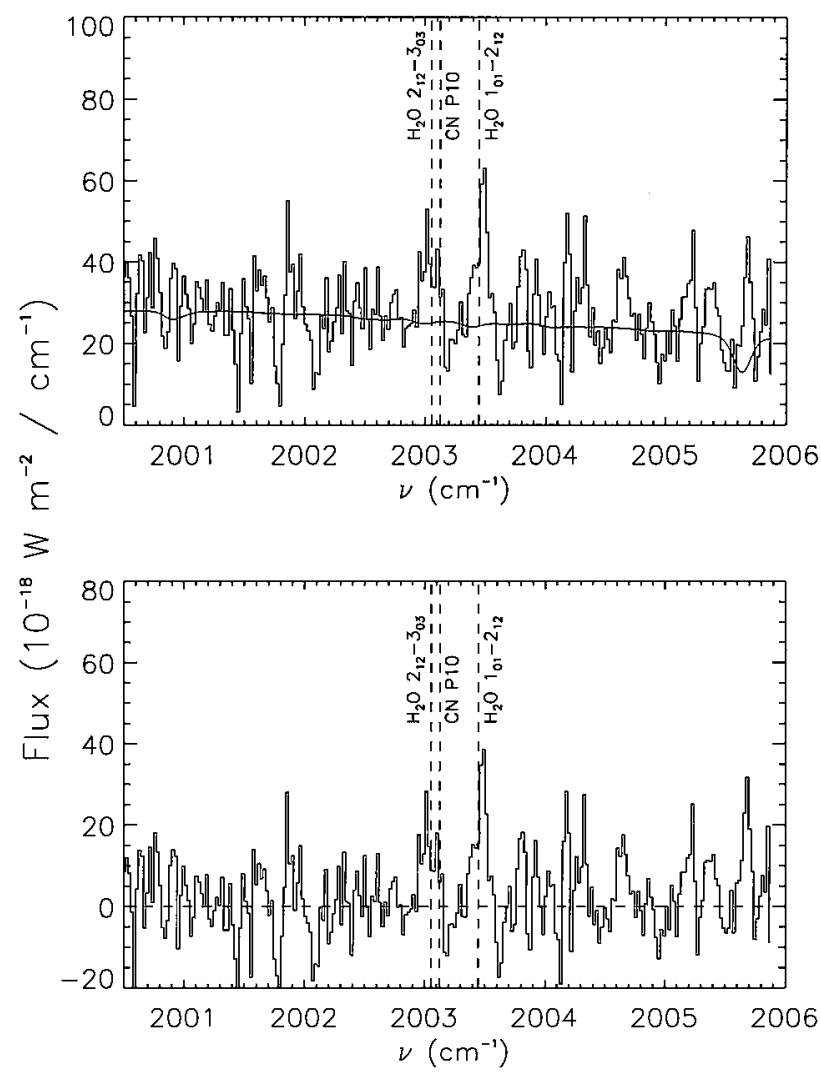

FIG . 2. The top panel shows a spectrum of Comet 21P/Giacobini-Zinner taken on UT 27 October 1998 using CSHELL at the NASA IRTF. The predicted locations of three cometary lines are shown by the dashed vertical lines. The solid curve is a model for the observed cometary continuum, including atmospheric absorption. Subtraction of the latter from the observed spectrum yields the emission spectrum shown in the bottom panel. The integrated flux in the stronger $\mathrm{H}_{2} \mathrm{O}$ line is detected with a signal-to-noise ratio of $\sim 6$.

range and is nearly coincident with one of the $\mathrm{H}_{2} \mathrm{O}$ lines (the $2_{12}-3_{03}$ line at $2002.999 \mathrm{~cm}^{-1}$ ). Our observing procedure (i.e., performing nods of only $10^{\prime \prime}$ ) discriminates against emission from photolysis products like $\mathrm{CN}$, and, indeed, the relevant feature in Fig. 2 is better aligned with the $\mathrm{H}_{2} \mathrm{O}$ line than with the $\mathrm{CN}$ line. However, as discussed in Section 2, the frequency scale for this spectrum is more uncertain than for our other cometary spectra, which makes us somewhat reluctant to rule out a possible contribution to this emission feature by $\mathrm{CN}$. Assuming that $T_{\text {rot }}$ is $50 \mathrm{~K}$, the production rate derived from the stronger, and uncontaminated, $\mathrm{H}_{2} \mathrm{O}$ line is $2.4 \times 10^{28}$ molecules $\mathrm{s}^{-1}$, which is similar to the value derived above for the $\mathrm{H}_{2} \mathrm{O}$ line in the $2-\mu \mathrm{m}$ band. Although the weaker $\mathrm{H}_{2} \mathrm{O}$ line in the $5-\mu \mathrm{m}$ band may be contaminated by $\mathrm{CN}$ emission, we note that its flux relative to the stronger line appears to be consistent with the theoretical value of $\sim 0.4$ at $50 \mathrm{~K}$.

Our primary objective on 26 October was to search for $\mathrm{CO}$ lines, but we targeted specific $\mathrm{CO}$ transitions that also allowed us to search for two relatively strong $\mathrm{H}_{2} \mathrm{O}$ lines: one in the $v_{1}-v_{2}$ band and one in the $v_{3}-v_{2}$ band. Both of these $\mathrm{H}_{2} \mathrm{O}$ lines 

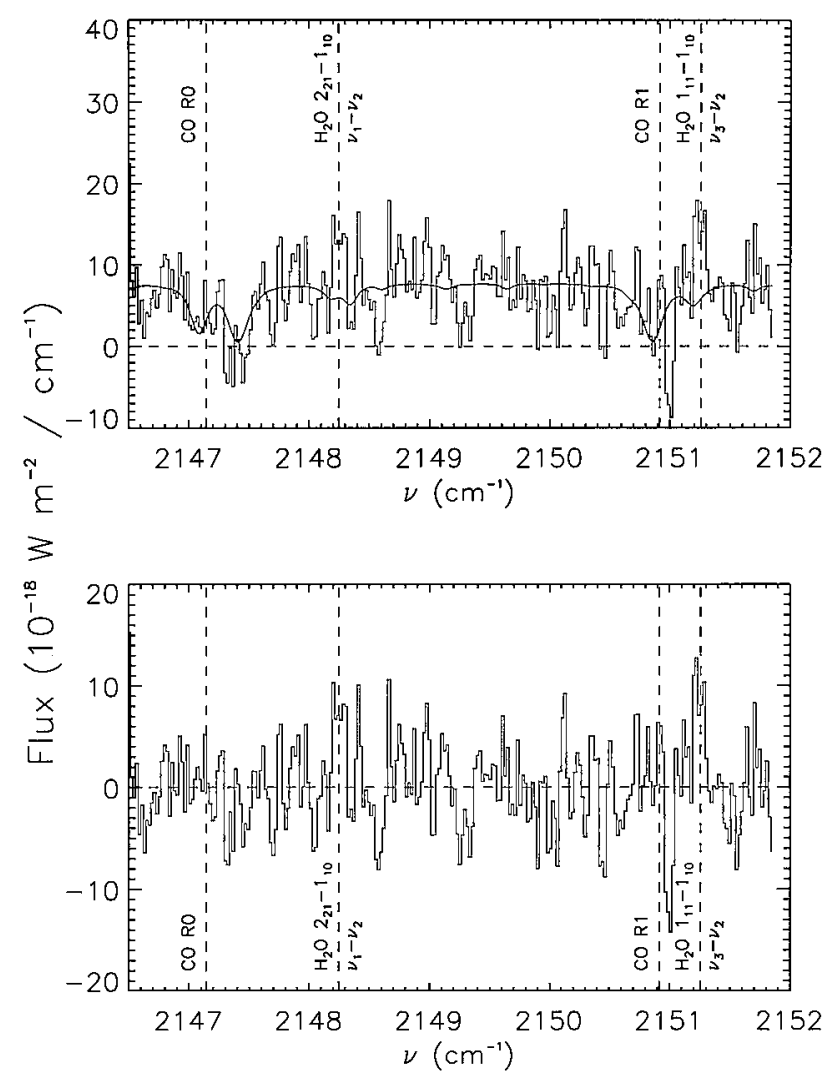

FIG. 3. The top panel shows a spectrum of Comet 21P/Giacobini-Zinner taken on UT 26 October 1998 using CSHELL at the NASA IRTF. The predicted locations of four cometary lines are shown by the dashed vertical lines. The solid curve is a model for the observed cometary continuum, including the atmospheric absorptions. Subtraction of the latter from the observed spectrum yields the emission spectrum shown in the bottom panel. The integrated flux in the stronger $\mathrm{H}_{2} \mathrm{O}$ line was detected with a signal-to-noise ratio of $\sim 8$. No significant cometary $\mathrm{CO}$ emission was detected.

appear to be present in our spectrum (Fig. 3), at their expected Doppler-shifted positions and with the expected spectral shape (i.e., a width of $\sim 5$ pixels for these observations, which were made with a $1^{\prime \prime}$ slit). However, the relative intensity of the two $\mathrm{H}_{2} \mathrm{O}$ lines is very different from the model g-factor ratio. The observed lines have a flux ratio of $\sim 1.3$ (i.e., have comparable intensities), whereas the predicted value is 5.3 for $T_{\text {rot }}=50 \mathrm{~K}$ and 3.4 for $T_{\text {rot }}=100 \mathrm{~K}$. For our Hale-Bopp observations at $r=1.1$ AU (Weaver et al. 1999), the observed intensity ratio was $\geq 4$, as we could not even clearly detect the $2_{21}-1_{10}$ line. Assuming that $T_{\text {rot }}$ is $50 \mathrm{~K}$, the flux in the $1_{11}-1_{10}$ implies a $\mathrm{H}_{2} \mathrm{O}$ production rate of $\sim 1.2 \times 10^{28}$ molecules $\mathrm{s}^{-1}$, whereas the flux in the $2_{21}-1_{10}$ line gives a $\mathrm{H}_{2} \mathrm{O}$ production rate of $\sim 5.3 \times 10^{28}$ molecules $\mathrm{s}^{-1}$. Interestingly, the average of these two values is similar to the $\mathrm{H}_{2} \mathrm{O}$ production rate derived earlier during our analysis of the $\mathrm{H}_{2} \mathrm{O}$ lines at other wavelengths. Perhaps the apparent inconsistency in the relative flux ratios of the $\mathrm{H}_{2} \mathrm{O}$ lines observed in the $\mathrm{CO}$ region is due to the poor signal-to-noise of the data.
In summary, our CSHELL observations of $\mathrm{H}_{2} \mathrm{O}$ lines in comet GZ indicate that $\mathrm{Q}_{\mathrm{H}_{2} \mathrm{O}}$ was $\sim 2-3 \times 10^{28}$ molecules s ${ }^{-1}$ during the entire period of our observations. UV observations of GZ during its 1985 apparition, at the same heliocentric distance as our 1998 observations, gave a peak value for $\mathrm{Q}_{\mathrm{H}_{2} \mathrm{O}}$ of $\sim 3.5-$ $5.4 \times 10^{28}$ molecules s $^{-1}$ (using data from McFadden et al. 1986 and Schleicher et al. 1987, but using updated vectorial density model parameters; the range reflects different choices for the $\mathrm{H}_{2} \mathrm{O}$ and $\mathrm{OH}$ velocities and lifetimes). Radio observations of $\mathrm{OH}$ in GZ give a $\mathrm{H}_{2} \mathrm{O}$ production rate of $5.5 \pm 0.4 \times 10^{28}$ molecules $\mathrm{s}^{-1}$ at $r \sim 1.1 \mathrm{AU}$ in 1985 (production rates from Gérard et al. 1988 were reevaluated using updated model parameters) and $5.6 \pm 0.4 \times 10^{28} \mathrm{mols}$ in 1998 (average of observations made between 26 October and 31 October; Biver 1999c). Thus, the 1998 IR value for $\mathrm{Q}_{\mathrm{H}_{2} \mathrm{O}}$ is 1.9-2.8 times smaller than the radio values, which are virtually identical in 1985 and 1998, and 1.2-2.7 times smaller than the 1985 UV values. Given the poor signal-to-noise ratio of the IR determinations of $\mathrm{Q}_{\mathrm{H}_{2} \mathrm{O}}$, one could possibly accept that they are consistent with both the 1998 radio value and the 1985 radio and UV values. Although a variety of physical mechanisms (e.g., seasonal effects and mantling) could produce differences in activity between the 1985 and 1998 apparitions of GZ, the data above indicate a remarkable consistency in $\mathrm{Q}_{\mathrm{H}_{2} \mathrm{O}}$ values for the two epochs.

\section{2. $\mathrm{CH}_{3} \mathrm{OH}$, the 3.43- $\mu \mathrm{m}$ Feature, $\mathrm{H}_{2} \mathrm{CO}$, PAHs, and the Continuum}

Figure 4 shows the CGS4 spectrum of GZ taken from the UKIRT on 29 October along with our estimate of the continuum contribution from cometary grains. The continuum was modeled as the sum of a scattered solar spectrum, which has a color temperature of $5800 \mathrm{~K}$, and a thermal component. Photometry of GZ between 4.7 and $20 \mu \mathrm{m}$ at $r=1$ AU in 1985 indicated that the thermal continuum was well-fit by a blackbody spectrum at $300 \mathrm{~K}$ (Hanner et al. 1992). However, the combination of UKIRT and IRTF data (see Fig. 5) indicates that a $300 \mathrm{~K}$ blackbody curve is too steep in the 3 - to $5-\mu \mathrm{m}$ region to match the spectrum. We found that $T \sim 380 \mathrm{~K}$ provides a much better fit to the data.

The estimated continuum was subtracted from the observed UKIRT spectrum to produce the "emission" spectrum displayed in Fig. 4. This figure also shows a model $\mathrm{CH}_{3} \mathrm{OH}$ spectrum that includes fluorescence in the $\mathrm{C}-\mathrm{H}$ stretch fundamental bands: $v_{2}$ (centered near $3.334 \mu \mathrm{m}$ ), $\nu_{9}$ (centered near $3.367 \mu \mathrm{m}$ ), and $\nu_{3}$ (centered near $3.516 \mu \mathrm{m}$ ). The fluorescence model for $\mathrm{CH}_{3} \mathrm{OH}$ includes collisional excitation of the rotational levels and vibrational pumping by solar infrared radiation (Bockelée-Morvan et al. 1994) and has been used to analyze the $\mathrm{CH}_{3} \mathrm{OH}$ contribution to the IR spectra of several other comets (BockeléeMorvan et al. 1995). Laboratory line assignments and intensities for the $\mathrm{C}-\mathrm{H}$ stretch fundamentals are currently incomplete, and the model uses symmetric top approximations for these. In addition, overtone and combination bands of $\mathrm{CH}_{3} \mathrm{OH}$ may also contribute significant emission in the 3.4- to $3.5-\mu \mathrm{m}$ region, 

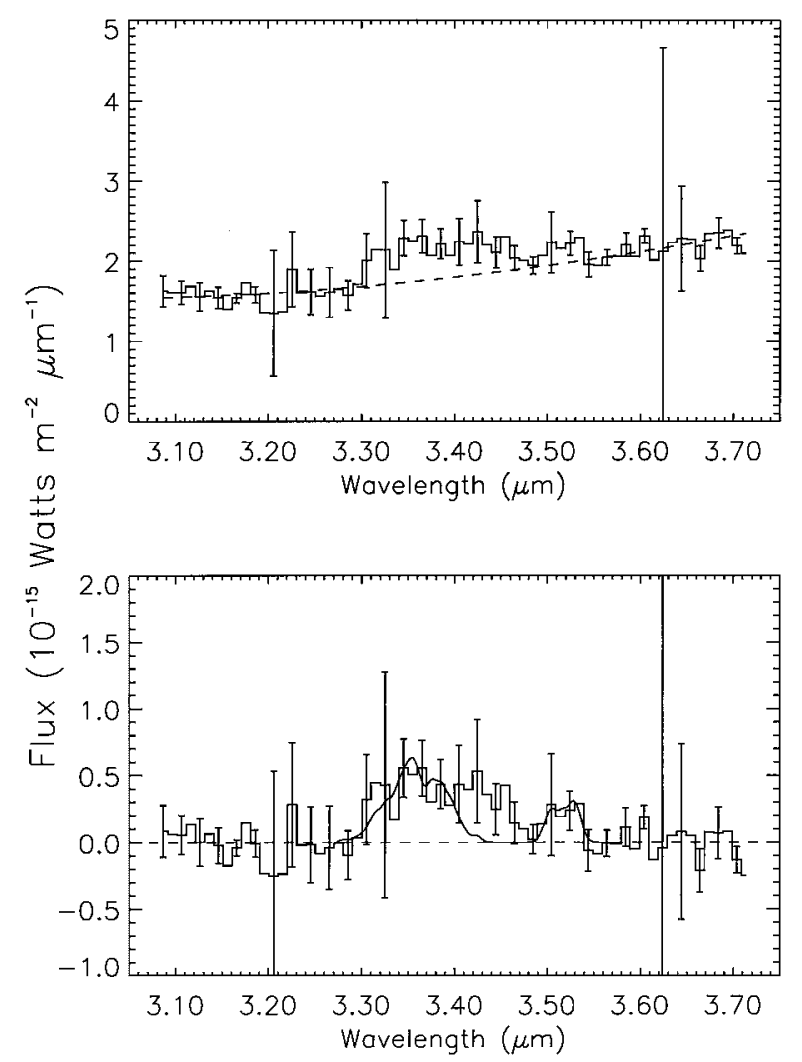

FIG . 4. The top panel shows the spectrum of Comet 21P/Giacobini-Zinner measured using CGS4 at UKIRT on 29 October 1998. The dashed curve is the best fit continuum, which consists of a scattered light component having a color temperature of $5800 \mathrm{~K}$ and a thermal component having a color temperature of $380 \mathrm{~K}$. Subtraction of this continuum from the observed spectrum yields the emission spectrum displayed in the bottom panel. The solid curve in the bottom panel is a model $\mathrm{CH}_{3} \mathrm{OH}$ fluorescence spectrum, which explains most of the observed emission. However, there is an additional emission feature near $3.43 \mu \mathrm{m}$.

and these bands are not included in the model. Yet the $\mathrm{CH}_{3} \mathrm{OH}$ production rates derived from the IR observations generally agree very well with the values derived from radio observations because the total strength of the $v_{3}$ band is well determined, and this band is usually the one used to derive $\mathrm{CH}_{3} \mathrm{OH}$ production rates. Assuming that all the residual emission near $3.52 \mu \mathrm{m}$ is due to the $\mathrm{CH}_{3} \mathrm{OH} \nu_{3}$ band, we derive a $\mathrm{CH}_{3} \mathrm{OH}$ production rate of $2.7 \pm 0.8 \times 10^{26}$ molecules s ${ }^{-1}$. Assuming that the $\mathrm{H}_{2} \mathrm{O}$ production rate is $2-3 \times 10^{28}$ molecules s${ }^{-1}$, the $\mathrm{CH}_{3} \mathrm{OH} /$ water abundance ratio is $0.9-1.4 \%$. Radio observations of $\mathrm{CH}_{3} \mathrm{OH}$ in GZ on 31 October from the James Cleark Maxwell Telescope (JCMT) give $\mathrm{Q}_{\mathrm{CH}_{3} \mathrm{OH}}=6.9 \pm 1.1 \times 10^{26}$ molecules s ${ }^{-1}$ (Biver, personal communication, assuming $\left.v=0.8 \mathrm{~km} \mathrm{~s}^{-1}\right)$. However, since the radio-derived value for $\mathrm{Q}_{\mathrm{H}_{2} \mathrm{O}}$ is also $\sim 2$ times larger than our value (see Section 3.1), we obtain similar $\mathrm{CH}_{3} \mathrm{OH}$ abundances (the radio value is $1.3 \pm 0.3 \%$ ). Thus, the IR and radio data give consistent $\mathrm{CH}_{3} \mathrm{OH}$ abundance ratios and demonstrate that the $\mathrm{CH}_{3} \mathrm{OH}$ abundance in $\mathrm{GZ}$ falls within the range of values observed in other comets (1-7\%; see Bockelée-Morvan 1997), albeit at the low end.
After subtraction of the model $\mathrm{CH}_{3} \mathrm{OH}$ spectrum from the CGS4 data, significant residual emission remains near $3.43 \mu \mathrm{m}$ (see Fig. 4). Fits to the spectra of other comets in this spectral region also showed a similar residual emission (Bockelée-Morvan et al. 1995, DiSanti et al. 1995). The residual flux correlates better with the $\mathrm{H}_{2} \mathrm{O}$ production rate than with the dust continuum in this sample, suggesting that it is of gaseous origin. The residual flux correlates even better with the $\mathrm{CH}_{3} \mathrm{OH}$ production rate. As discussed above, the spectrum of $\mathrm{CH}_{3} \mathrm{OH}$ is not well known in this region and there is a possibility that all or part of this residual emission may be due to overtone and/or combination bands of $\mathrm{CH}_{3} \mathrm{OH}$ itself (see Bockelée-Morvan et al. 1995 for a more detailed discussion). Here we simply examine how the residual flux in GZ compares to that in other comets.

Following the approach of Brooke et al. (1991), we computed the quantity $F^{*}=F(980 / \rho) \Delta^{2}$, where $\rho$ is the effective aperture radius in kilometers and $\Delta$ is the geocentric distance in AU. $F^{*}$ is proportional to the production rate of the unknown species and can be compared to the values computed for other comets (see Bockelée-Morvan et al. 1995). We find that $F^{*} / Q_{\mathrm{H}_{2} \mathrm{O}}$ in comet GZ (0.09-0.14 $\left.\left[10^{-15} \mathrm{~W} \mathrm{~m}^{-2} / 10^{29} \mathrm{~s}^{-1}\right]\right)$ is a factor of $\sim 3-4$ smaller than the value indicated by a global fit to the heliocentric dependence of this quantity $(\sim 0.4$ in the same units, at $r \sim$ $1 \mathrm{AU})$ for all of the comets discussed by Bockelée-Morvan et al. (1995). The value of $F^{*} / \mathrm{Q}_{\mathrm{CH}_{3} \mathrm{OH}}$ in comet $\mathrm{GZ}, \sim 0.1\left[10^{-15} \mathrm{~W}\right.$ $\left.\mathrm{m}^{-2} / 10^{27} \mathrm{~s}^{-1}\right]$, is only $\sim 40 \%$ smaller than the global average for all comets and is essentially identical to the value determined

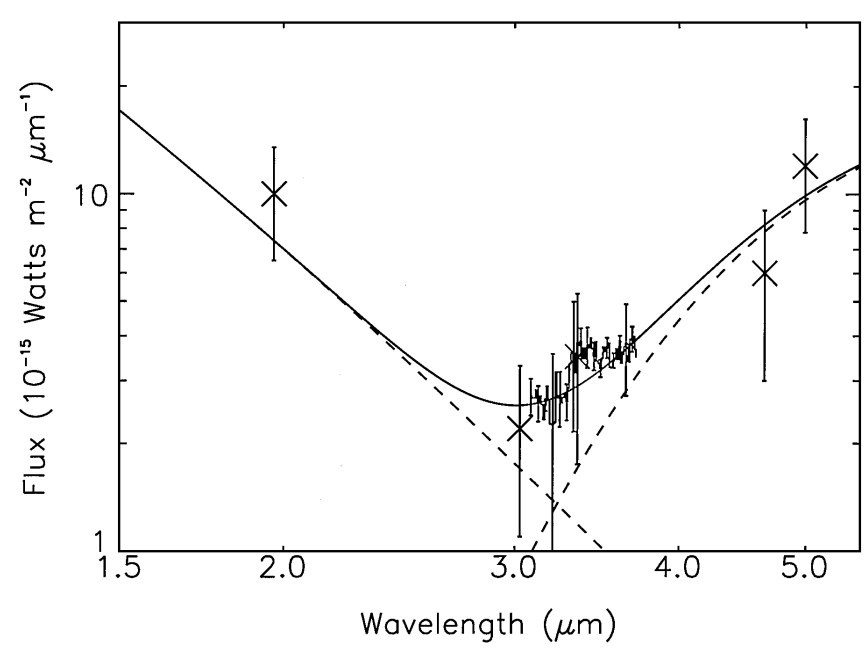

FIG . 5. The UKIRT CGS4 spectrum (histogram with error bars) of Comet 21P/Giacobini-Zinner taken on 29 October 1998 is plotted together with the average continuum levels derived from the IRTF CSHELL spectra $(\times$ symbols $)$ taken on (from left to right) 29 October, 28 October, 25 October, 26 October, and 27 October. All displayed fluxes have been normalized to an effective aperture size of $2^{\prime \prime} \times 4^{\prime \prime}$. The solid curve is the best fit continuum, assuming that the scattered solar continuum has a color temperature of $5800 \mathrm{~K}$ (left dashed curve) and that the thermal continuum has a color temperature of $380 \mathrm{~K}$ (right dashed curve). 
for 1P/Halley ( $\sim 0.09$ in the same units) at $r \sim 1$ AU. ${ }^{12}$ Thus, the abundances of both $\mathrm{CH}_{3} \mathrm{OH}$ and the species responsible for the $3.43-\mu \mathrm{m}$ emission appear to be at the low end of the range of values observed in other comets.

The CGS4 spectrum can also be used to place a constraint on the $\mathrm{H}_{2} \mathrm{CO}$ abundance in GZ. The $v_{1}$ and $v_{5}$ bands of $\mathrm{H}_{2} \mathrm{CO}$ are centered at 3.594 and $3.517 \mu \mathrm{m}$, respectively, and $\sim 25 \%$ of the total emission in these bands lies between 3.57 and $3.60 \mu \mathrm{m}$. There is no residual emission in the latter wavelength region after subtraction of the continuum, and we have used the $3 \sigma$ noise level to place an upper limit on the $\mathrm{H}_{2} \mathrm{CO}$ production rate of $1.5 \times 10^{26}$ molecules $\mathrm{s}^{-1}$, assuming that $\mathrm{H}_{2} \mathrm{CO}$ is a parent molecule. This translates into an upper limit on the $\mathrm{H}_{2} \mathrm{CO}$ abundance of $\sim 0.5-0.8 \%$. If we instead assume that the $\mathrm{H}_{2} \mathrm{CO}$ comes from an extended source whose scalelength is $7000 \mathrm{~km}$, we calculate an upper limit to the $\mathrm{H}_{2} \mathrm{CO}$ abundance of $\sim 5-8 \%$. Both of these limits on the $\mathrm{H}_{2} \mathrm{CO}$ abundance in $\mathrm{GZ}$ are considerably larger than what is typically observed in comets: $\sim 0.2 \%$ if a parent species and $\sim 1 \%$ if from an extended source (BockeléeMorvan 1997). However, our limit on $\mathrm{H}_{2} \mathrm{CO}$ production as a parent molecule is well below the value derived by Mumma and Reuter (1989) from the Vega infrared spectroscopy of Comet 1P/Halley $(\sim 4 \%)$.

We note that the CGS4 spectrum of GZ shows no evidence for any excess emission near $3.28 \mu \mathrm{m}$. Since polycyclic aromatic hydrocarbon (PAH) molecules can radiate at this wavelength, there is a strong interest in identifying and characterizing any emission there. Excess emission at $3.28 \mu \mathrm{m}$ has been detected in several comets, but its strength relative to $\mathrm{H}_{2} \mathrm{O}$ and $\mathrm{CH}_{3} \mathrm{OH}$ production seems to be highly variable among comets (see Bockelée-Morvan et al. 1995). The absence of any significant excess emission in the spectrum of comet GZ near $3.28 \mu \mathrm{m}$ is certainly not unique.

In Fig. 5 we display all of the cometary continuum measurements made during our investigation of GZ. As discussed above, the continuum consists of a thermal component having a color temperature of $\sim 380 \mathrm{~K}$ and a scattered solar component. Only the CSHELL point near $2 \mu \mathrm{m}$ and the CGS4 spectrum were obtained on the same day, yet all of the data are remarkably consistent. ${ }^{13}$ Apparently the continuum emission from GZ was fairly constant (to within $\sim 50 \%$ ) during the entire period of our observations. The 1985 optical and UV observations of GZ (Schleicher et al. 1987) also showed little day-to-day variability.

\section{3. $\mathrm{C}_{2} \mathrm{H}_{6}$}

One of the biggest surprises during the observations of Comet Hyakutake (C/1996 B2) was the detection, using CSHELL, of

\footnotetext{
12 This result is due to the relatively large $\mathrm{CH}_{3} \mathrm{OH}$ abundance of $\sim 5 \%$ assumed for Halley at $r=1 \mathrm{AU}$.

${ }^{13}$ We have multiplied the CGS4 spectrum by a factor of 1.67 to scale its flux from what was observed in a $1^{\prime \prime} .2 \times 2^{\prime \prime} .4$ aperture to what would be expected in a $2^{\prime \prime} \times 4^{\prime \prime}$ aperture. Similarly, the CSHELL point near $4.67 \mu \mathrm{m}$ was multiplied by 1.6 to convert from a $1^{\prime \prime} \times 4^{\prime \prime}$ aperture to a $2^{\prime \prime} \times 4^{\prime \prime}$ aperture.
}

abundant $\mathrm{C}_{2} \mathrm{H}_{6}$ (Mumma et al. 1996). The Q subbranches of this molecule are very strong and many fall at wavelengths where there is good transmittance through the terrestrial atmosphere, so sensitive searches for $\mathrm{C}_{2} \mathrm{H}_{6}$ are possible. The comparable $\mathrm{C}_{2} \mathrm{H}_{6}$ abundances, relative to $\mathrm{H}_{2} \mathrm{O}$, in Comets Hyakutake and HaleBopp ( 0.4\%, Weaver et al. 1999, Kim et al. 2000) proved that Hyakutake was not unique in its $\mathrm{C}_{2} \mathrm{H}_{6}$ abundance and suggests that $\mathrm{C}_{2} \mathrm{H}_{6}$ might be similarly abundant in all "normal" comets. On 25 October we conducted a deep CSHELL search for the ${ }^{\mathrm{r}} \mathrm{Q}_{0}$ and ${ }^{\mathrm{r}} \mathrm{Q}_{1}$ subbranches in the $v_{7} \mathrm{C}_{2} \mathrm{H}_{6}$ band in comet GZ. In addition, the CGS4 spectrum taken on 29 October provided another probe of $\mathrm{C}_{2} \mathrm{H}_{6}$ emission.

The CSHELL spectrum (Fig. 6) does not show any evidence for $\mathrm{C}_{2} \mathrm{H}_{6}$ emission, despite the long exposure time employed (170 $\mathrm{min}$ ). Even the cometary continuum was only marginally detected, as we were observing in a region near the minimum of the continuum flux (see Fig. 5). We calculated $\mathrm{C}_{2} \mathrm{H}_{6} \mathrm{~g}$ factors assuming that the populations of the ground-state rotational levels were in thermal equilibrium with $T_{\text {rot }}=50 \mathrm{~K}$ (Kim et al. 2000). The lack of emission in the ${ }^{\mathrm{r}} \mathrm{Q}_{0}$ subbranch provides the most

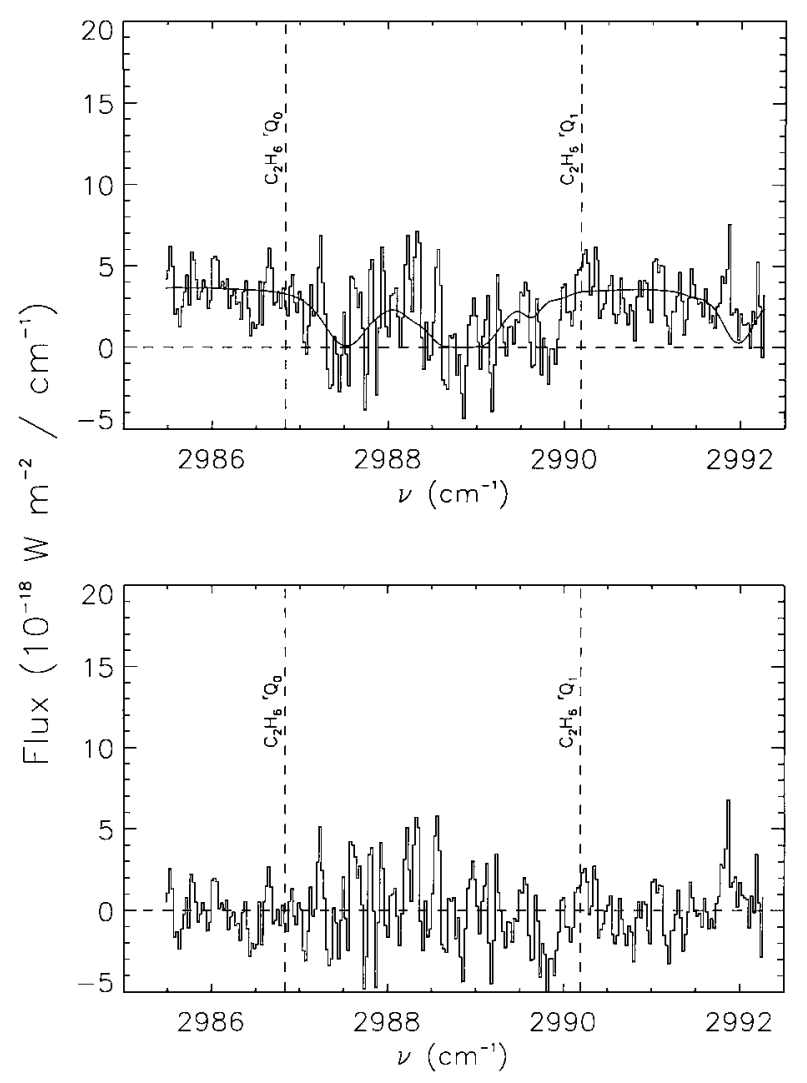

FIG. 6. The top panel shows a spectrum of Comet 21P/Giacobini-Zinner taken on UT 29 October 1998 using CSHELL at the NASA IRTF. The solid curve is a model for the observed cometary continuum, including the atmospheric absorptions. Subtraction of the latter from the observed spectrum yields the emission spectrum shown in the bottom panel. The predicted locations of two $\mathrm{C}_{2} \mathrm{H}_{6}$ subbranches $\left({ }^{\mathrm{r}} \mathrm{Q}_{0}\right.$ and $\left.{ }^{\mathrm{r}} \mathrm{Q}_{1}\right)$ are shown by the dashed vertical lines, but no $\mathrm{C}_{2} \mathrm{H}_{6}$ emission was detected. 
sensitive limit on the $\mathrm{C}_{2} \mathrm{H}_{6}$ production rate, $\mathrm{Q}_{\mathrm{C}_{2} \mathrm{H}_{6}} \leq 1.6 \times 10^{25}$ molecules $\mathrm{s}^{-1}(3 \sigma)$. Assuming that the $\mathrm{H}_{2} \mathrm{O}$ production rate was $\sim 2-3 \times 10^{28}$ molecules s ${ }^{-1}$, we find that $\mathrm{C}_{2} \mathrm{H}_{6} / \mathrm{H}_{2} \mathrm{O} \leq 0.05-$ $0.08 \%$, which implies that $\mathrm{C}_{2} \mathrm{H}_{6}$ was depleted in $\mathrm{GZ}$ by a factor of at least 5-8 compared to Comets Hale-Bopp and Hyakutake. This depletion factor is similar to that found for the $C_{2}$ and $C_{3}$ abundances in GZ from ground-based optical observations: the $\mathrm{C}_{2} / \mathrm{OH}$ and $\mathrm{C}_{3} / \mathrm{OH}$ ratios are about one-tenth of the "typical" cometary value, and the $\mathrm{C}_{2} / \mathrm{CN}$ and $\mathrm{C}_{3} / \mathrm{CN}$ ratios are about onefifth of the typical value (A'Hearn et al. 1995). Thus, our result on $\mathrm{C}_{2} \mathrm{H}_{6}$ strengthens the conclusion that "depleted" comets are underabundant in volatile carbon-chain molecules. However, we note the possibility that more complex carbon chain molecules might still be present in the nuclei of "carbon-chain depleted" comets; if those molecules have relatively high sublimation temperatures and are not released in gaseous form in the coma, then they would be difficult to detect even if they were relatively abundant. The organic residues produced during laboratory irradiation experiments on cometary ice analogs (see Greenberg 1982) are possible examples.

Mumma et al. (2000) detected emission in the ${ }^{\mathrm{r}} \mathrm{Q}_{0}$ and ${ }^{\mathrm{P}} \mathrm{Q}_{1}$ subbranches of $\mathrm{C}_{2} \mathrm{H}_{6}$ during CSHELL observations of GZ during the first week of October 1998, but their $\mathrm{C}_{2} \mathrm{H}_{6}$ production rate of $\sim 8 \times 10^{25}$ molecules $\mathrm{s}^{-1}$ is $\sim 5$ times larger than our $3 \sigma$ upper limit. Based on GZ's behavior during the 1985 apparition, gas production rates in early October 1998 were expected to be $\sim 50 \%$ smaller than at the end of the month, ${ }^{14}$ making the discrepancy between our results and those of Mumma et al. even more puzzling. Mumma et al. also detected $\mathrm{CO}$ emission in GZ (see further discussion below) but did not detect any $\mathrm{H}_{2} \mathrm{O}$ lines, whereas we detected five $\mathrm{H}_{2} \mathrm{O}$ lines but no $\mathrm{CO}$ emission. Both groups generally searched for the same lines, so changes in the molecular excitation are unlikely to explain these discrepancies. If we use their value for $\mathrm{Q}_{\mathrm{C}_{2} \mathrm{H}_{6}}$ and our value for $\mathrm{Q}_{\mathrm{H}_{2} \mathrm{O}}$, then $\mathrm{C}_{2} \mathrm{H}_{6} / \mathrm{H}_{2} \mathrm{O} \sim 0.3-0.4 \%$ and there would be essentially no depletion of $\mathrm{C}_{2} \mathrm{H}_{6}$. A factor of five depletion in the $\mathrm{C}_{2} \mathrm{H}_{6} / \mathrm{H}_{2} \mathrm{O}$ abundance in early October would require $\mathrm{Q}_{\mathrm{H}_{2} \mathrm{O}}=7.2 \times 10^{28}$ molecules $\mathrm{s}^{-1}$ at that time, which is inconsistent with the nondetection of IR $\mathrm{H}_{2} \mathrm{O}$ emission by Mumma et al. We have not yet been able to resolve the above discrepancies.

Our CGS4 spectrum of GZ can also be used to place an upper limit on the $\mathrm{C}_{2} \mathrm{H}_{6}$ abundance. Emission from the $\mathrm{C}_{2} \mathrm{H}_{6} v_{7}$ band would appear as a broad feature (FWHM $\sim 0.04 \mu \mathrm{m}$ ) centered at a wavelength near $\sim 3.35 \mu \mathrm{m}$ in the CGS4 spectrum. After subtraction of the modeled cometary continuum and $\mathrm{CH}_{3} \mathrm{OH}$ emissions, the CGS4 spectrum shows no significant residual emission at $3.35 \mu \mathrm{m}$. Adopting a band $\mathrm{g}$ factor of $7.3 \times 10^{-4}$ photons s ${ }^{-1}$ molecule $^{-1}$ (Kim et al. 2000), we find that the the $3 \sigma$ limit on the residual flux translates into an upper limit on the $\mathrm{C}_{2} \mathrm{H}_{6}$ production rate of $\sim 8.8 \times 10^{25}$ molecules $\mathrm{s}^{-1}$. The latter is

\footnotetext{
${ }^{14}$ Indeed, the total visual magnitude was $\sim 0.5$ mags brighter at the end of October compared to early October.
}

$\sim 5$ times larger than the upper limit derived from our CSHELL data. $^{15}$

Although the CGS4 spectrum does not provide a very constraining limit on $\mathrm{C}_{2} \mathrm{H}_{6}$ production in Comet $\mathrm{GZ}$, moderate resolution spectra exist for a number of comets from which $\mathrm{C}_{2} \mathrm{H}_{6}$ abundance limits can be estimated. We used the spectra of the seven comets discussed in Bockelée-Morvan et al. (1995) and assumed that all of the residual emission they assigned to a broad feature centered at $3.35 \mu \mathrm{m}$ after subtraction of a model of $\mathrm{CH}_{3} \mathrm{OH}$ emission is due to $\mathrm{C}_{2} \mathrm{H}_{6}$. The results are summarized in Table III.

First, we note that Comet 1P/Halley shows evidence for compositional heterogeneity as two of its spectra (on 25 April 1986 and 19-20 May 1986) have residuals near $3.35 \mu \mathrm{m}$ that could be explained by a relative $\mathrm{C}_{2} \mathrm{H}_{6}$ abundance of $\sim 1-2 \%$, while the $\mathrm{C}_{2} \mathrm{H}_{6}$ abundance must be at least an order of magnitude smaller to explain the lack of any residual emission on 28 March 1986. Compositional heterogeneity was previously invoked to explain variations in the $\mathrm{H}_{2} \mathrm{CO}$ abundance in Halley (Mumma and Reuter 1989). The residual emissions near $3.35 \mu \mathrm{m}$ in Comets Levy (C/1990 K1), Okazaki-Levy-Rudenko (C/1989 XIX), Wilson (C/1986 P1), and Bradfield (C/1987 P1) apparently require $\mathrm{C}_{2} \mathrm{H}_{6}$ abundances equal to or greater than the $\mathrm{C}_{2} \mathrm{H}_{6}$ abundances in Comets Hyakutake (C/1996 B2) and Hale-Bopp (C/1995 O1). However, for these four comets the residual centered near $3.35 \mu \mathrm{m}$ is less than half the total residual emission between 3.2 and $3.5 \mu \mathrm{m}$, after subtraction of the predicted $\mathrm{CH}_{3} \mathrm{OH}$ contribution, indicating that something other than $\mathrm{C}_{2} \mathrm{H}_{6}$ must also be contributing significantly and that the $\mathrm{C}_{2} \mathrm{H}_{6}$ production rates quoted in Table III should probably be regarded as upper limits. There is evidence for $\mathrm{C}_{2} \mathrm{H}_{6}$ depletion in the spectra of Comets Austin (C/1989 X1) and 23P/Brorsen-Metcalf, although one spectrum of the Brorsen-Metcalf may require a small amount of $\mathrm{C}_{2} \mathrm{H}_{6}$ emission.

Table III also gives the estimated $\mathrm{C}_{2}$ and $\mathrm{C}_{3}$ abundances for the various comets. Although the IR spectra indicate that comets 21P/Brorsen-Metcalf and Austin (C/1989 X1) may be depleted in $\mathrm{C}_{2} \mathrm{H}_{6}$, both have "normal" $\mathrm{C}_{2}$ and $\mathrm{C}_{3}$ abundances. All of the other comets in the table, except GZ, have normal $\mathrm{C}_{2}$ and $\mathrm{C}_{3}$ abundances, so the data presented in this paper do not provide any convincing evidence for a correlation between $\mathrm{C}_{2} \mathrm{H}_{6}$ depletion and the $\mathrm{C}_{2}$ and $\mathrm{C}_{3}$ depletions. We also do not find any correlation of the strength of the $3.35-\mu \mathrm{m}$ emission band with dynamical origin.

Although our analysis indicates that $\mathrm{C}_{2} \mathrm{H}_{6}$ emission is probably a significant contributor to the excess flux observed in moderate resolution spectra of some comets near $3.35 \mu \mathrm{m}$, we must also recognize the limitations of the approach used here. In

\footnotetext{
${ }^{15}$ Note that if our model overestimates the $\mathrm{CH}_{3} \mathrm{OH}$ fluorescence in the $v_{2}$ and $\nu_{9}$ bands, as was suggested by Bockelée-Morvan et al. (1995), then $\mathrm{Q}_{\mathrm{C}_{2} \mathrm{H}_{6}}$ derived from the CGS4 spectrum could be larger than the value given here and quoted in Table III. The same is true for the limits on $\mathrm{Q}_{\mathrm{C}_{2} \mathrm{H}_{6}}$ derived for the other comets discussed later.
} 
TABLE III

Limits on $\mathrm{C}_{2} \mathrm{H}_{6}$ A bundances in Comets

\begin{tabular}{|c|c|c|c|c|c|c|c|c|c|c|c|}
\hline Comet & $\mathrm{r}^{a}$ & $\begin{array}{l}\Delta^{a} \\
\mathrm{U})\end{array}$ & $\begin{array}{l}\mathrm{FOV}^{b} \\
(\operatorname{arcsec})\end{array}$ & $\begin{array}{c}\mathrm{Q}_{\mathrm{H}_{2} \mathrm{O}}^{c} \\
\left(10^{28} \mathrm{~s}^{-1}\right)\end{array}$ & $\begin{array}{c}\text { Flux at } 3.35 \mu \mathrm{m}^{d} \\
\left(10^{-16} \mathrm{~W} \mathrm{~m}^{-2}\right)\end{array}$ & $\begin{array}{c}\mathrm{Q}_{\mathrm{C}_{2} \mathrm{H}_{6}}^{e} \\
\left(10^{26} \mathrm{~s}^{-1}\right)\end{array}$ & $\begin{array}{c}\mathrm{C}_{2} \mathrm{H}_{6} / \mathrm{H}_{2} \mathrm{O} \\
(\%)\end{array}$ & $\begin{array}{c}\mathrm{C}_{2} \mathrm{H}_{6} / \mathrm{CH}_{3} \mathrm{OH}^{f} \\
(\%)\end{array}$ & $\begin{array}{c}\mathrm{C}_{2} / \mathrm{OH}^{g} \\
(\%)\end{array}$ & $\begin{array}{c}\mathrm{C}_{3} / \mathrm{OH}^{g} \\
(\%)\end{array}$ & $\begin{array}{c}\mathrm{C}_{2} / \mathrm{CN}^{g} \\
(\%)\end{array}$ \\
\hline 1P/Halley & 1.11 & 0.62 & 5 & 50 & $\leq 9.3$ & $\leq 6.8$ & $\leq 0.14$ & $\leq 3$ & 0.77 & 0.078 & 150 \\
\hline 1P/Halley & 1.56 & 0.65 & 2.5 & 20 & 16 & 45 & 2.3 & 120 & 0.5 & 0.05 & 130 \\
\hline 1P/Halley & 1.90 & 1.37 & 1.37 & 30 & 2.2 & 37 & 1.2 & 110 & 0.5 & 0.05 & 130 \\
\hline C/1986 P1 (Wilson) & 1.31 & 1.09 & 1.35 & 11 & 3.6 & 23 & 2.1 & 55 & 0.14 & 0.02 & 54 \\
\hline C/1987 P1 (Bradfield) & 0.89 & 0.97 & 1.35 & 7 & 2.5 & 6.7 & 0.96 & 28 & 0.69 & 0.069 & 120 \\
\hline 23P/Brorsen-Metcalf & 0.95 & 0.62 & 1.35 & 7 & $\leq 0.90$ & $\leq 1.7$ & $\leq 0.24$ & $\leq 13$ & 0.40 & 0.028 & 140 \\
\hline 23P/Brorsen-Metcalf & 0.61 & 0.80 & 1.35 & 9 & $\leq 2.1$ & $\leq 2.2$ & $\leq 0.25$ & $\leq 6$ & 0.44 & 0.026 & 130 \\
\hline 23P/Brorsen-Metcalf & 0.48 & 1.20 & 1.35 & 16 & 2.8 & 2.9 & 0.18 & 6 & 0.56 & 0.023 & 140 \\
\hline C/Okazaki-Levy-Rudenko & 0.66 & 0.65 & 1.35 & 6 & 4.6 & 4.6 & 0.76 & 23 & 0.6 & 0.02 & 72 \\
\hline C/1989 X1 (Austin) & 0.97 & 0.31 & 2.5 & 6 & $\leq 3.3$ & $\leq 1.8$ & $\leq 0.29$ & $\leq 9$ & 0.44 & 0.026 & 130 \\
\hline C/1990 K1 (Levy) & 1.37 & 0.43 & 2.5 & 25 & 8.8 & 13 & 0.52 & 81 & 0.30 & 0.021 & 130 \\
\hline C/1996 B2 (Hyakutake) & 1.06 & 0.11 & 0.67 & 23 & - & 10 & 0.43 & 27 & 0.48 & 0.035 & 150 \\
\hline C/1995 O1 (Hale-Bopp) & 1.05 & 1.46 & 0.80 & 500 & - & 170 & 0.34 & 13 & 0.79 & 0.10 & 130 \\
\hline 21P/Giacobini-Zinner & 1.10 & 0.95 & 0.96 & $2-3$ & $\leq 0.21$ & $\leq 0.88$ & $\leq 0.3-0.5$ & $\leq 30$ & 0.04 & 0.003 & 20 \\
\hline
\end{tabular}

${ }^{a} r, \Delta$ : helocentric and geocentric distances, respectively.

${ }^{b}$ FOV: equivalent circular aperture radius.

${ }^{c} \mathrm{Q}_{\mathrm{H}_{2} \mathrm{O}}$ : from Bockelée-Morvan et al. (1995), except for Hyakutake (Dello Russo et al., personal communication Hale-Bopp (Weaver et al. 1999), and GZ (this work).

${ }^{d}$ Flux @ $3.35 \mu \mathrm{m}$ : from Bockelée-Morvan et al. (1995), except for GZ (this work).

${ }^{e} \mathrm{Q}_{\mathrm{C}_{2} \mathrm{H}_{6}}$ : assumes residual emission at $3.35 \mu \mathrm{m}$ is due to the $\mathrm{C}_{2} \mathrm{H}_{6} \nu_{7}$ band; for Comets Hyakutake and Hale-Bopp, $\mathrm{C}_{2} \mathrm{H}_{6}$ production rates are based on clear identifications of several subbranches in the $\mathrm{C}_{2} \mathrm{H}_{6} v_{7}$ band (Mumma et al. 1996, Weaver et al. 1999, Kim et al. 2000) but the values are preliminary.

${ }^{f} \mathrm{C}_{2} \mathrm{H}_{6} / \mathrm{CH}_{3} \mathrm{OH}$ : using $\mathrm{Q}_{\mathrm{CH}_{3} \mathrm{OH}}$ from Bockelée-Morvan et al. (1995), except for Hyakutake (Biver et al. 1999b), Hale-Bopp (Biver et al. 1999a), and GZ (this work); for $\mathrm{GZ}, \mathrm{C}_{2} \mathrm{H}_{6} / \mathrm{H}_{2} \mathrm{O} \leq 0.05-0.08 \%$ and $\mathrm{C}_{2} \mathrm{H}_{6} / \mathrm{CH}_{3} \mathrm{OH} \leq 5.9 \%$ from our high resolution data (see Table II).

${ }^{g} \mathrm{C}_{2} / \mathrm{OH}, \mathrm{C}_{3} / \mathrm{OH}$, andC $2 / \mathrm{CN}$ : from A'Hearn et al. (1995), Schleicher et al. (1991), Schleicher et al. (1998), and Schleicher (personal communication).

particular, the $\mathrm{C}_{2} \mathrm{H}_{6}$ abundance limits derived from these moderate resolution spectra could be wrong, if our $\mathrm{CH}_{3} \mathrm{OH}$ fluorescence model incorrectly predicts the emission in the 3.35$\mu \mathrm{m}$ region. Again, see the discussion in Bockelée-Morvan et al. (1995) for a detailed discussion of the limitations of the $\mathrm{CH}_{3} \mathrm{OH}$ fluorescence model. There is no substitute for high spectral resolution observations that clearly detect individual subbranches in the $v_{7}$ band of $\mathrm{C}_{2} \mathrm{H}_{6}$, and our statements above regarding cometary $\mathrm{C}_{2} \mathrm{H}_{6}$ abundances must be considered preliminary. Further progress must await future high spectral resolution detections of $\mathrm{C}_{2} \mathrm{H}_{6}$ in other comets and/or improvements in modeling $\mathrm{CH}_{3} \mathrm{OH}$ fluorescence.

\section{4. $\mathrm{CO}$}

The sublimation temperature of pure $\mathrm{CO}$ ice in outer solar nebula conditions is $\sim 24 \mathrm{~K}$ (see Yamamoto 1985), which makes it one of the most sensitive indicators of a comet's evolutionary history. This molecule has now been detected in eight comets, with abundances ranging from a few percent to $~ 30 \%$ (Feldman et al. 1997, Bockelée-Morvan 1997). However, this sample of comets is highly biased towards very bright, long-period objects. Prior to the 1998 observations of GZ, Schwassmann-Wachmann 1 (SW1) was the only Jupiter-family comet ${ }^{16}$ for which there had

\footnotetext{
${ }^{16}$ Defined as comets having a Tisserand parameter with respect to Jupiter of $T_{J}>2$; see Levison and Duncan 1997.
}

been a definite detection of $\mathrm{CO}$, but SW1 never passes within 5.7 AU of the Sun. At least one short-period comet, 103P/Hartley 2 with a period of only 6.3 years, does not show any evidence for $\mathrm{CO}$ in its nucleus to a $3 \sigma$ limit of $\sim 1 \%$ relative to $\mathrm{H}_{2} \mathrm{O}$ (Weaver et al. 1994). Thus, searches for $\mathrm{CO}$ in other short-period comets may provide important insights into the ability of comets to retain this very volatile species that is so ubiquitous throughout the universe.

We searched for fluorescent emission from $\mathrm{GZ}$ in the $\mathrm{CO}(1,0)$ fundamental band near $4.67 \mu \mathrm{m}$ on 26 October. As previously discussed, we used the $1^{\prime \prime}$-wide slit and beam-switched every $10 \mathrm{~s}$ to provide good discrimination between the cometary and terrestrial $\mathrm{CO}$ features. The average spectrum is displayed in Fig. 3 and does not show any evidence for $\mathrm{CO}$ emission in the $\mathrm{R} 0$ or R1 lines. From the $3 \sigma$ upper limits on the $\mathrm{CO}$ fluxes at the top of the atmosphere, and using $\mathrm{CO} g$ factors calculated assuming that $T_{\text {rot }}=50 \mathrm{~K}$, we derive an upper limit on the $\mathrm{CO}$ production rate of $\mathrm{Q}_{\mathrm{CO}} \leq 6.5 \times 10^{26}$ molecules $\mathrm{s}^{-1}$, assuming that $\mathrm{CO}$ is a parent molecule. The corresponding abundance ratio limit is $\sim 2-3 \%$. For $T_{\text {rot }}=100 \mathrm{~K}$, the abundance ratio limit is $\sim 4-6 \%$. Our limit on CO production in GZ is similar to the estimated abundance of $\mathrm{CO}$ in the nucleus of Comets 1P/Halley (3.5\%, Eberhardt 1998), Bradfield (C/1979 Y1) (3.5\%, Feldman et al. 1997), and Levy (C/1990 K1) (4.1-8.4\%, Feldman et al. 1997), and is significantly lower than the estimated $\mathrm{CO}$ abundances in comets Hyakutake (C/1996 B2) ( 14\%, McPhate et al. 1996; 20\%, Biver et al. 1999b; Mumma et al. 1996 
derived a preliminary value of $7 \%)$ and Hale-Bopp ( $\sim 10 \%$ at $r \sim 1 \mathrm{AU}$, according to Weaver et al. 1999 and DiSanti et al. 1999; $\sim 23 \%$ at $r \sim 1 \mathrm{AU}$, according to Biver et al. 1999a). The $\mathrm{CO}$ abundance limit for GZ is somewhat larger than the abundance ratio observed in Comet Austin (C/1989 X1) (1.7\%, Feldman et al. 1997) and the upper limits derived for 103P/Hartley $2(\leq 1.2 \%$, Weaver et al. 1994) and Shoemaker-Levy (C/1991 T2) ( $\leq 1.8 \%$, Feldman et al. 1997). Thus, CO in GZ is depleted relative to some comets, but GZ could have a $\mathrm{CO}$ abundance that is comparable to other comets considered "normal" in the A'Hearn et al. (1995) compilation. ${ }^{17}$

Unfortunately, the atmospheric transmittances at the Doppler-shifted CO R0 and R1 line positions for GZ were only $\sim 0.45$ and $\sim 0.25$, respectively, which means that our observation would have been up to four times more sensitive if the absolute value of the geocentric radial velocity had been larger than $\sim 15 \mathrm{~km} \mathrm{~s}^{-1}$. Our observations demonstrate that $\mathrm{CO}$ can be probed to very sensitive abundance limits, even in relatively faint comets, if the comets have reasonably large Doppler shifts.

As mentioned earlier, Mumma et al. (2000) detected CO emission in GZ during their observations in early October. However,

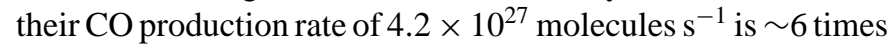
larger than our $3 \sigma$ upper limit from late October and $\sim 4$ times larger than the $3 \sigma$ upper limit derived from radio observations in later October (Biver et al. 1999c). We searched unsuccessfully for CO Fourth Positive Group emission in a UV spectrum taken five days postperihelion during the 1985 IUE observations of GZ (see McFadden et al. 1987) and derive $\mathrm{Q}_{\mathrm{CO}} \leq 2 \times 10^{27}$ molecules s ${ }^{-1}(3 \sigma)$ at $r=1.03 \mathrm{AU}$. If we use the Mumma et al. value for $\mathrm{Q}_{\mathrm{CO}}$ and our value for $\mathrm{Q}_{\mathrm{H}_{2} \mathrm{O}}$, then $\mathrm{CO} / \mathrm{H}_{2} \mathrm{O} \sim 14-21 \%$, which is very high for a short-period comet. As stated earlier, we find the discrepancies between our results and those of Mumma et al. puzzling and have not found a viable way to resolve them.

\section{5. $\mathrm{HCN}$ and $\mathrm{C}_{2} \mathrm{H}_{2}$}

On 28 October we searched for lines in the $v_{3}$ band of $\mathrm{C}_{2} \mathrm{H}_{2}$ and for lines in the $\nu_{3}$ band of $\mathrm{HCN}$ at a wavelength near $3 \mu \mathrm{m}$. The average spectrum for this region is displayed in Fig. 7. There is a marginally significant (signal-to-noise ratio of $\sim 4$ ) emission feature present near the expected position of the $\mathrm{HCN}$ P3 line, but we do not regard this as a definite detection of HCN because the HCN P2 and P4 lines were not detected even though they should have comparable intensities. Emission in the $v_{1}$ band of $\mathrm{NH}_{2}$ could possibly explain the slight excess emission near $3302 \mathrm{~cm}^{-1}$, but we do not claim to detect any significant cometary emissions in this spectrum.

We adopt the measured integrated flux at the top of the atmosphere for the HCN P3 line as an upper limit on the brightness of this line, from which we derive $\mathrm{Q}_{\mathrm{HCN}} \leq 5.4 \times 10^{25}$ molecules s $^{-1}$,

\footnotetext{
${ }^{17} \mathrm{C} / 1989 \mathrm{X} 1,103 \mathrm{P} / \mathrm{Hartley} 2$, and C/1991 T2 have typical $\mathrm{C}_{2}, \mathrm{C}_{3}$, and CN abundances.
}

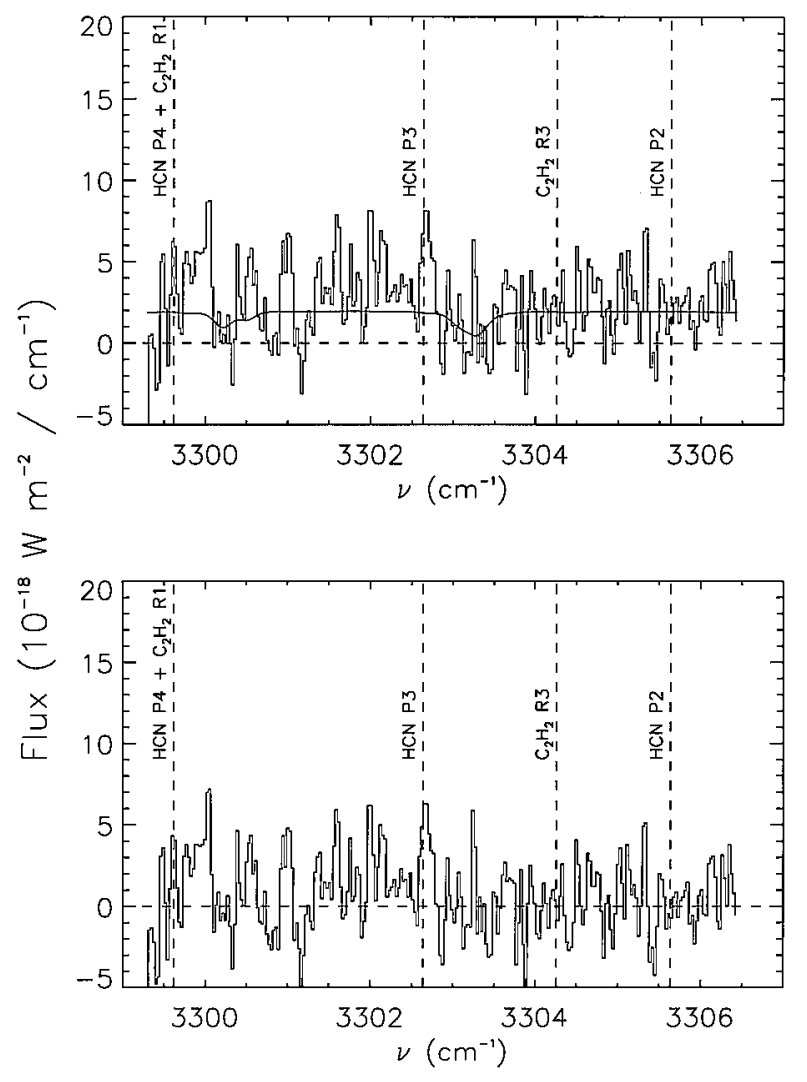

FIG. 7. The top panel shows a spectrum of Comet 21P/Giacobini-Zinner taken on UT 28 October 1998 using CSHELL at the NASA IRTF. The solid curve is a model for the cometary continuum, including atmospheric absorption. Subtraction of the latter from the observed spectrum yields the emission spectrum shown in the bottom panel. The dashed vertical lines show the predicted locations of three $\mathrm{HCN}$ lines and two $\mathrm{C}_{2} \mathrm{H}_{2}$ lines, but we do not claim any definite detections in this spectrum.

assuming $T_{\text {rot }}=50 \mathrm{~K}$ and using the $\mathrm{HCN}$ excitation model described in Crovisier (1987). HCN was clearly detected during radio observations of GZ on 30 October and 2 November, and the derived production rate was $\sim 3 \times 10^{25}$ molecules s $^{-1}$ (Biver, personal communication). Thus, our null IR result is consistent with the radio detection of HCN. Using the radio-derived (i.e., from $\mathrm{OH}$ ) value for $\mathrm{Q}_{\mathrm{H}_{2} \mathrm{O}}$ of $5.3 \pm 0.7 \times 10^{28}$ molecules s ${ }^{-1}$, the radio observation indicates that $\mathrm{GZ}$ had a relative $\mathrm{HCN}$ abundance of $\sim 0.05-0.07 \%$, which falls just below the "normal" range of $\sim 0.1-0.2 \%$ (see Bockelée-Morvan 1997).

The $\mathrm{C}_{2} \mathrm{H}_{2}$ molecule was first reported in comets during CSHELL observations of Comet Hyakutake (C/1996 B2) (Brooke et al. 1996) and was subsequently observed in Comet Hale-Bopp (C/1995 O1) (Weaver et al. 1999, Magee-Sauer et al. 1999). The derived $\mathrm{C}_{2} \mathrm{H}_{2}$ abundance was $0.3-0.9 \%$ in Hyakutake, depending on the adopted values of $T_{\text {rot }}$ and $\mathrm{Q}_{\mathrm{H}_{2} \mathrm{O}}$, and was $\sim 0.09 \%$ in Hale-Bopp. ${ }^{18}$ Assuming that the CSHELL spectrum of GZ between 3304 and $3305 \mathrm{~cm}^{-1}$ is pure noise, we

\footnotetext{
${ }^{18}$ This is a preliminary value from Weaver et al. (1999).
} 
estimate that the $3 \sigma$ limit on the flux of the $\mathrm{C}_{2} \mathrm{H}_{2} \mathrm{R} 3$ line ${ }^{19}$ is $5.9 \times 10^{-19} \mathrm{~W} \mathrm{~m}^{-2}$. This translates into a production rate limit of $8.3 \times 10^{25}$ molecules $\mathrm{s}^{-1}$ and an upper limit on the $\mathrm{C}_{2} \mathrm{H}_{2}$ abundance of $\sim 0.3-0.4 \%$. As the latter is essentially identical to the observed $\mathrm{C}_{2} \mathrm{H}_{2}$ abundance in Hyakutake, our CSHELL observation does not provide much of a constraint on any possible $\mathrm{C}_{2} \mathrm{H}_{2}$ depletion in comet $\mathrm{GZ}$.

\section{SUMMARY}

We detected five rovibrational lines in three different vibrational bands of $\mathrm{H}_{2} \mathrm{O}$ during high spectrtal resolution observations of comet Giacobini-Zinner in late October 1998. Although each line was detected at low signal-to-noise, the appearance of multiple lines at the expected Doppler-shifted positions, their spectral widths, and their absolute intensities provide additional evidence that the identifications are correct. We derive a $\mathrm{H}_{2} \mathrm{O}$ production rate of $\sim 2-3 \times 10^{28}$ molecules $\mathrm{s}^{-1}$ during the period of our observations, which was roughly 25 days preperihelion. Our $\mathrm{H}_{2} \mathrm{O}$ production rate is $\sim 2$ times smaller than the value derived from nearly simultaneous 1998 radio observations of $\mathrm{OH}$ and is also $\sim 2$ times smaller than the values derived from radio and $\mathrm{UV}$ observations of $\mathrm{OH}$ made $\sim 25$ days preperihelion in 1985.

During moderate resolution observations of GZ on 29 October 1998, we detected excess emission between 3.3 and $3.6 \mu \mathrm{m}$. We demonstrate that the bulk of this emission can be accounted for by fluorescence from $\mathrm{CH}_{3} \mathrm{OH}$. The $\mathrm{CH}_{3} \mathrm{OH}$ production rate was $\sim 2.7 \pm 0.8 \times 10^{26}$ molecules $\mathrm{s}^{-1}$, which corresponds to an abundance ratio of $0.9-1.4 \%$ relative to $\mathrm{H}_{2} \mathrm{O}$. Our $\mathrm{CH}_{3} \mathrm{OH}$ relative abundance is in excellent agreement with the value derived from radio observations made in late-October, but our absolute $\mathrm{CH}_{3} \mathrm{OH}$ production rate is about one-half the radio value. Both the IR and radio results demonstrate that GZ has a "normal" $\mathrm{CH}_{3} \mathrm{OH}$ abundance, although at the low end of the observed range.

$\mathrm{CH}_{3} \mathrm{OH}$ alone cannot account for all the excess cometary emission between 3.3 and $3.6 \mu \mathrm{m}$; additional residual emission appears centered near $3.43 \mu \mathrm{m}$. The latter feature in GZ is similar in its central wavelength and spectral shape to an unidentified spectral feature observed in the IR spectra of several other comets. The ratio of the intensity of the $3.43-\mu \mathrm{m}$ feature to the $\mathrm{H}_{2} \mathrm{O}$ production rate in $\mathrm{GZ}$ is a factor of $\sim 3-$ 4 smaller than the average value observed in other comets. However, the strength of the 3.43- $\mu \mathrm{m}$ feature compared to the $\mathrm{CH}_{3} \mathrm{OH}$ production rate in $\mathrm{GZ}$ is similar to the average ratio in other comets. Thus, both the $\mathrm{CH}_{3} \mathrm{OH}$ abundance and the abundance of the species producing the 3.43- $\mu \mathrm{m}$ feature, which could be $\mathrm{CH}_{3} \mathrm{OH}$, are near the low end of the ranges observed in other comets.

\footnotetext{
${ }^{19}$ This is expected to be the strongest $\mathrm{C}_{2} \mathrm{H}_{2}$ line in the R-branch for $T_{\mathrm{rot}}=$ $50 \mathrm{~K}$, using the excitation model of Crovisier (1987).
}

We did not detect any excess emission from GZ near $3.28 \mu \mathrm{m}$. Emission has been detected at this wavelength in a few comets and may possibly be associated with polycyclic aromatic hydrocarbons, although no definite identification has been made.

We did not detect $\mathrm{C}_{2} \mathrm{H}_{6}, \mathrm{C}_{2} \mathrm{H}_{2}, \mathrm{CO}$, or $\mathrm{HCN}$ during our high spectral resolution observations of GZ. We find that $\mathrm{C}_{2} \mathrm{H}_{6}$ is depleted in GZ by a factor of at least 5 compared to comets Hyakutake and Hale-Bopp; this depletion is similar to the observed underabundance of $\mathrm{C}_{2}$ and $\mathrm{C}_{3}$ in GZ relative to "typical" cometary abundances. Thus, our result lends support to the hypothesis that volatile carbon-chain molecules are depleted in GZ. Our upper limit on the $\mathrm{C}_{2} \mathrm{H}_{2}$ abundance $(\leq 0.2-0.4 \%)$ is also consistent with this hypothesis, but is not as constraining as the $\mathrm{C}_{2} \mathrm{H}_{6}$ upper limit. Our upper limit on the $\mathrm{CO}$ abundance in the nucleus of GZ demonstrates that this molecule is depleted relative to its abundance in comets Hyakutake (C/1996 B2) and Hale-Bopp (C/1995 O1), but is not necessarily depleted relative to the values derived for several other comets. Our upper limit on the $\mathrm{HCN}$ abundance $(\leq 0.2-0.3 \%)$ is consistent with radio results obtained at nearly the same time as our IR observations and which show that GZ has an $\mathrm{HCN}$ abundance at the low end of the "normal" range.

Our program on Comet 21P/Giacobini-Zinner demonstrates that ground-based near-IR spectroscopy of relatively faint comets can provide sensitive probes of several parent volatiles (e.g., $\mathrm{H}_{2} \mathrm{O}, \mathrm{C}_{2} \mathrm{H}_{6}$, and $\mathrm{C}_{2} \mathrm{H}_{2}$ ) that cannot be observed by any other ground-based technique. Further improvements in IR telescopes and instrumentation in the future should allow for abundance measurements of these molecules in many short-period comets.

Note added in proof: Mumma et al. (2000) multiplied their raw production rates for comet GZ by a factor of 2 to correct for the effects of seeing, whereas we generally used a larger extraction aperture and did not make a similar correction. This difference could potentially account for about half of the discrepancy in production rates derived by the two groups, as discussed in the body of the paper. If our observations sampled a different vent on the nucleus than the one sampled by Mumma et al., perhaps our different results indicate that the nucleus of comet GZ is compositionally heterogeneous.

\section{ACKNOW LED GMENTS}

We thank Bill Golisch and Dave Griep for their excellent technical support of these observations. We thank N. Dello Russo for fruitful discussions on $\mathrm{H}_{2} \mathrm{O}$ g factors, $\mathrm{N}$. Biver for providing radio production rates for $\mathrm{GZ}$ prior to publication, and $\mathrm{D}$. Schleicher for providing updated $\mathrm{C}_{2}, \mathrm{C}_{3}$, and $\mathrm{CN}$ abundances. We also thank the two referees, Humberto Campins and Dave Schleicher, for their helpful comments. H.A.W., G.C., and T.Y.B. gratefully acknowledge financial support for this work from NASA through Grant NAG5-7186 from the Planetary Astronomy Program. S.J.K. gratefully acknowledges support from Grant 96-07-02-03-01-3 from the Korean Science and Engineering Foundation. UKIRT is operated by the Joint Astronomy Centre on behalf of the United Kingdom Particle Physics and Astronomy Research Council. 


\section{REFERENCES}

A'Hearn, M. F., R. L. Millis, D. G. Schleicher, D. J. Osip, and P. V. Birch 1995. The ensemble properties of comets: Results from narrowband photometry of 85 comets, 1976-1992. Icarus 118, 223-270.

A'Hearn, M. F., D. G. Schleicher, P. D. Feldman, R. L. Millis, and D. T. Thompson 1984. Comet Bowell 1980b. Astron. J. 89, 579-591.

Biver, N., and 23 colleagues 1999a. Long term evolution of the outgassing of Comet Hale-Bopp from radio observations. Earth, Moon, Planets, in press.

Biver, N., D. Bockelée-Morvan, and P. Colom, et al. 1997. Evolution of the outgassing of Comet Hale-Bopp (C/1995 O1) from radio observations. Science 275, 1915-1918.

Biver, N., D. Bockelée-Morvan, J. Crovisier, J. K. Davies, H. E. Matthews, J. E. Wink, H. Rauer, P. Colom, W. R. F. Dent, D. Despois, R. Moreno, G. Paubert, D. Jewitt, and M. Senay 1999b. Spectroscopic monitoring of Comet C/1996 B2 (Hyakutake) with the JCMT and IRAM telescopes. Astron. J. 118, 1850-1872.

Biver, N., J. Crovisier, J. K. Davies, D. Despois, D. C. Lis, D. Bockelée-Morvan, P. Colom, E. Gérard, H. E. Matthews, and G. Paubert 1999c. Coordinated radio observations of Comet 21P/Giacobini-Zinner in October-November 1998. In abstracts book for Asteroids, Comets, Meteors meeting, 26-30 July 1999, Cornell University, Ithaca.

Bockelée-Morvan, D. 1987. A model for the excitation of water in comets. Astron. Astrophys. 181, 169-181.

Bockelée-Morvan, D. 1997. Cometary volatiles. In Molecules in Astrophysics: Probes and Processes (E. F. Van Dishoeck, Ed.), pp. 219-235. Kluwer Academic, Dordrecht.

Bockelée-Morvan, D., and J. Crovisier 1987. The role of water in the thermal balance of the coma. In Symposium on the Diversity and Similarity of Comets, Brussels, Belgium, 6-9 April 1987, ESA SP-278, 235240.

Bockelée-Morvan, D., and J. Crovisier 1988. The nature of the $2.8 \mu \mathrm{m}$ emission feature in comets. Astron. Astrophys. 216, 278-283.

Bockelée-Morvan, D., T. Brooke, and J. Crovisier 1995. On the origin of the 3.2- to 3.6- $\mu \mathrm{m}$ emission features in comets. Icarus 116, 18-39.

Bockelée-Morvan, D., J. Crovisier, P. Colom, and D. Despois 1994. The rotational lines of methanol in Comets Austin $1990 \mathrm{~V}$ and Levy 1990 XX. Astron. Astrophys. 287, 647-665.

Bockelée-Morvan, D., J. Crovisier, and E. Gérard 1990. Retrieving the coma gas expansion velocity in P/Halley, Wilson (1987 VII) and several other comets from the $18-\mathrm{cm} \mathrm{OH}$ line shapes. Astron. Astrophys. 238, 382400.

Brooke, T. Y., A. T. Tokunaga, and R. F. Knacke 1991. Detection of the $3.4 \mu \mathrm{m}$ emission feature in Comets P/Brorsen-Metcalf and Okazaki-Levy-Rudenko (1989r) and an observation summary. Astron. J. 101, 268-278.

Brooks, T. Y., A. T. Tokunaga, H. A. Weaver, J. Crovisier, D. Bockelée-Morvan, and D. Crisp 1996. Detection of acetylene in the infrared spectrum of Comet Hyakutake. Nature 383, 606-608.

Clough, S. A., F. X. Kneizys, E. P. Shettle, and G. P. Anderson 1986. Atmospheric radiance and transmittance: FASCOD2. In Proc. Sixth Conf. Atmos. Radiation, American Met. Soc., pp. 141-144.

Cochran, A. L., and E. S. Barker 1987. Comet Giacobini-Zinner: A normal comet? Astron. J. 92, 239-243.

Crovisier, J. 1987. Rotational and vibrational synthetic spectra of linear parent molecules in comets. Astron. Astrophys. Suppl. Ser. 68, 223-258.

Crovisier, J., and Th. Encrenaz 1983. Infrared fluorescence of molecules in comets-The general synthetic spectrum. Astron. Astrophys. 126, 170-182.

Crovisier, J., K. Leech, D. Bockelée-Morvan, T. Y. Brooke, M. S. Hanner, B. Altieri, H. U. Keller, and E. Lellouch 1997. The spectrum of Comet Hale-Bopp (C/1995 O1) observed with the Infrared Space Observatory at 2.9 astronomical units from the Sun. Science 275, 1904-1907.
DiSanti, M. A., M. J. Mumma, N. Dello Russo, K. Magee-Sauer, R. Novak, and T. W. Retting 1999. Identification of two sources of carbon monoxide in Comet Hale-Bopp. Nature 399, 662-665.

DiSanti, M. A., M. J. Mumma, T. R. Geballe, and J. K. Davies 1995. Systematic observations of methanol and other organics in Comet P/Swift-Tuttle: Discovery of new spectral structure at $3.42 \mu \mathrm{m}$. Icarus 116, 1-17.

Eberhardt, P. 1998. Volatiles, isotopes, and origin of comets. In Asteroids, Comets, Meteors '96: Proceedings of the 10th COSPAR Colloquium (A.-C. Levasseur-Regourd and M. Fulchignoni, Eds.). Pergamon-Elsevier, Oxford, in press.

Feldman, P. D., M. C. Festou, G.-P. Tozzi, and H. A. Weaver 1997. The $\mathrm{CO}_{2} / \mathrm{CO}$ abundance ratio in $1 \mathrm{P} /$ Halley and several other comets observed by IUE and HST. Astrophys. J. 475, 829-834.

Gérard, E., D. Bockelée-Morvan, G. Bourgois, P. Colom, and J. Crovisier 1988. Observations of the $\mathrm{OH}$ radio lines in Comet P/Giacobini-Zinner 1985 XII. Astron. Astrophys. Suppl. Ser. 74, 485-495.

Greenberg, J. M. 1982. What are comets made of-A model based on interstellar dust. In Comets (L. L. Wilkening, Ed.), pp. 131-163. Univ. of Arizona Press, Tucson.

Greene, T. P., A. T. Tokunaga, D. W. Toomey, and J. S. Carr 1993. CSHELL: A high spectral resolution 1-5 micron cryogenic echelle spectrograph for the IRTF. Proc. SPIE 1946, 313-324.

Hanner, M. S., G. J. Veeder, and A. T. Tokunaga 1992. The dust coma of Comet $\mathrm{P} /$ Giacobini-Zinner in the infrared. Astron. J. 104, 386-393.

Hayward, T. L., and G. L. Grasdalen 1987. Infrared images of comets. I. P/Giacobini-Zinner (1985e). Astron. J. 94, 1339-1347.

Kim, S. J., H. A. Weaver, and G. Chin 2000. The infrared excitation of $\mathrm{C}_{2} \mathrm{H}_{6}$ in comets. Icarus, submitted.

Levison, H. F., and M. J. Duncan 1997. From the Kuiper belt to Jupiter-family comets: The spatial distribution of ecliptic comets. Icarus 127, 13-32.

Magee-Sauer, K., M. J. Mumma, M. A. DiSanti, N. Dello Russo, and T. W. Rettig 1999. Infrared spectroscopy of the $v_{3}$ band of hydrogen cyanide in Comet C/1995 Hale-Bopp. Icarus 142, 498-508.

McFadden, L. A., and 10 colleagues 1987 . Ultraviolet spectrophotometry of Comet Giacobini-Zinner during the ICE encounter. Icarus 69, 329-337.

McPhate, J. B., P. D. Feldman, H. A. Weaver, M. F. A'Hearn, G.-P. Tozzi, and M. C. Festou 1996. Ultraviolet CO emission in Comet C/1996 B2 (Hyakutake). Bull. Am. Astron. Soc. 28, 0929.

Mumma, M. J., and D. C. Reuter 1989. On the identification of formaldehyde in Halley's comet. Astrophys. J. 344, 940-948.

Mumma, M. J., M. A. DiSanti, N. Dello Russo, M. Fomenkova, K. MageeSauer, C. D. Kaminski, and D. X. Xie 1996. Detection of abundant ethane and methane, along with carbon monoxide and water, in Comet C/1996 B2 Hyakutake: Evidence for interstellar origin. Science 272, 1310-1314.

Mumma, M. J., M. A. DiSanti, N. Dello Russo, K. Magee-Sauer, and T. W. Rettig 2000. Detection of CO and ethane in Comet 21P/Giacobini-Zinner: Evidence for variable chemistry in the outer solar nebula. Astrophys. J. Lett., submitted.

Mumma, M. J., H. A. Weaver, and H. P. Larson 1987. The ortho-para ratio of water vapor in Comet P/Halley. Astron. Astrophys. 187, 419-424.

Partidge, H., and D. W. Schwenke 1997. The determination of an accurate isotope dependent potential energy surface for water from extensive $a b$ initio calculations and experimental data. J. Chem. Phys. 106, 4618-4639.

Rothman, L. S., and 13 colleagues 1992. The HITRAN molecular database: Editions of 1991 and 1992. J. Quant. Spect. Rad. Transfer 48, 469-507.

Schleicher, D. G., R. L. Millis, and P. V. Birch 1987. Photometric observations of Comet P/Giacobini-Zinner. Astron. Astrophys. 187, 531-538.

Schleicher, D. G., R. L. Millis, and P. V. Birch 1998. Narrowband photometry of Comet P/Halley: Variation with heliocentric distance, season, and solar phase angle. Icarus 132, 397-417. 
Schleicher, D. G., R. L. Millis, D. J. Osip, and P. V. Birch 1991. Comet Levy (1990c) - Groundbased photometric results. Icarus 94, 511-523.

Sekanina, Z. 1985. Precession model for the nucleus of periodic Comet Giacobini-Zinner. Astron. J. 90, 827-845.

Telesco, C. M., R. Decher, C. Baugher, H. Campins, D. Mozurkewich, H. A. Thronson, D. P. Cruikshank, H. B. Hammel, S. Larson, and Z. Sekanina 1986. Thermal-infrared and visual imaging of Comet Giacobini-Zinner. Astrophys. J. 310, L61-L65.

Tokunaga, A. 1999. Infrared astronomy. In Astrophysical Quantities (A. Cox, Ed.), 4th ed. Springer-Verlag, Berlin, in press.
Weaver, H. A. and M. J. Mumma 1984. Infrared molecular emissions from comets. Astrophys. J. 276, 782-797. [Erratum: Astrophys. J. 285, 872]

Weaver, H. A., T. Y. Brooke, G. Chin, S. J. Kim, D. Bockelée-Morvan, and J. K. Davies 1999. Infrared spectroscopy of Comet Hale-Bopp. Earth, Moon, Planets, in press.

Weaver, H. A., P. D. Feldman, J. B. McPhate, M. F. A'Heaarn, C. Arpigny, and T. E. Smith 1994. Detection of CO Cameron band emission in Comet P/Hartley 2 (1991 XV) with the Hubble Space Telescope. Astrophys. J. 422, 374-380.

Yamamoto, T. 1982. Evaluation of infrared line emission from constituent molecules of cometary nuclei. Astron. Astrophys. 109, 326-330. 